\title{
Les dieux des autres : entre « démons » et «idoles »
}

Il est maintenant temps d'explorer un troisième domaine d'usage de la catégorie du démoniaque spécifique à la Bible hébraïque et à la Septante : celui de la définition des divinités étrangères. Même s'il n'y a souvent aucune différence significative entre la désignation des dieux des autres peuples ('élîm/

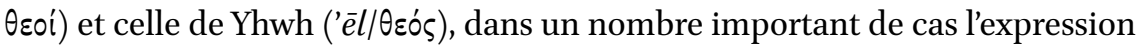
« démons » est utilisée de manière dérogatoire pour indiquer les dieux étrangers, notamment lorsqu'ils sont présentés en opposition ouverte au dieu d'Israël. De nombreuses raisons invitent à analyser cette thématique en détail. Tout d'abord, c'est dans ce contexte qu'apparaît une des attestations du mot šédîm dans la Bible hébraïque, et il s'agit de sa seule occurrence dans le Pentateuque ${ }^{1}$. C'est ici une occasion précieuse d'atteindre un niveau émique dans l'analyse, puisque nous avons accès à une réflexion menée par les anciens eux-mêmes sur leurs propres catégories religieuses. En second lieu, l'importance du rôle joué par la LXX dans la construction «démonologique» des autres dieux est indéniable. Non seulement elle a prolongé une représentation qui était propre à la Bible hébraïque en plusieurs contextes, comme par exemple dans le livre des Odes et celui de Baruch, mais elle a également constitué l'arrière-plan sur lequel s'est appuyée la représentation des dieux des autres comme démons. Cette représentation est mise en avant par le Nouveau Testament, notamment dans un fameux passage de Paul qui associe l'idolâtrie avec la table préparée pour les démons (1 Co 10,20-21 $)^{2}$. Effectivement, et comme nous le verrons en détail par la suite, nous devons davantage aux traducteurs grecs pour ce qui est du développement d'un lien entre la notion de démon et celle d'idole. Enfin, la démonisation des dieux étrangers est un phénomène qui n'est pas complètement absent des cultures environnantes, notamment de la culture grecque qui nous intéresse tout particulièrement ici : le sujet stimule donc une démarche comparatiste ainsi qu'une interrogation sur les spécificités du phénomène biblique.

Comme je l'ai déjà dit, le texte le plus important du corpus est représenté par un passage de Deut 32. Ce chapitre, connu également comme « Chant de Moïse » (Deut 32,1-43), contient l'un des deux discours prononcés par Moïse à la veille de sa mort, après avoir mis par écrit la Loi (Deut 31) et avant que

1 Voir supra, p. 8o.

2 Mais voir également Act 17,18; 1 Tim 4,1; Ap 9,20.

(C) ANNA ANGELINI, 2021 | DOI:10.1163/9789004468474_008

This is an open access chapter distributed under the terms of the CC BY-NC-ND 4.o licensenge Ange lini - 9789004468474 
son peuple, guidé par Josué, ne traverse le Jourdain pour entrer dans la terre de Canaan (Deut $32,48-52)^{3}$. Du point de vue narratif, ce texte occupe une place cruciale à l'intérieur du Deutéronome, mais également à l'intérieur du Pentateuque dans son ensemble. Par ses paroles, Moïse résume l'histoire d'Israël en remontant à ses origines mythiques, tout en annonçant son destin futur. Le discours est centré sur le contraste entre, d'une part, la fidélité et la générosité de Yhwh et, d'autre part, l'infidélité du peuple qui se manifeste d'abord et avant tout dans l'adoration des autres dieux, et qui a pour conséquence le déchaînement de la colère du dieu patron. Ces divinités, décrites

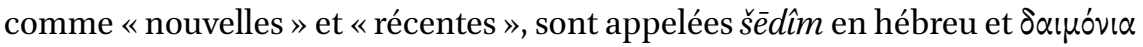
en grec $^{4}$ :

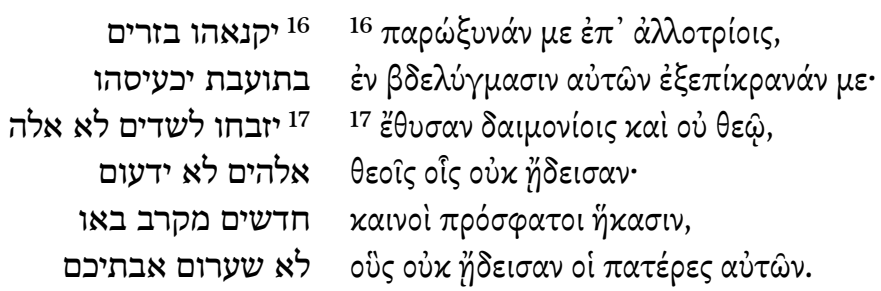

${ }^{16}$ Ils l'ont rendu jaloux par des cultes étrangers, par des abominations ils l'ont provoqué $(k s)$

${ }^{17}$ Ils ont sacrifié aux démons (šédîm), qui ne sont pas Dieu (lō"ělōah),

divinités qu'ils ne connaissaient pas, nouvelles, elles sont arrivées récemment, leurs pères ne les craignaient pas.
16 Ils me provoquèrent par des cultes étrangers, dans leurs abominations ils me remplirent d'amertume.

${ }^{17}$ Ils firent des sacrifices à des démons et non à Dieu, à des dieux, qu'ils n'avaient pas connus, des (dieux) nouveaux, récents sont arrivés, que leurs pères ne connaissaient pas.

Ces deux versets sont reproduits fidèlement en grec dans la deuxième des Odes où l'intégralité du chant est attribué au roi Salomon (Odes 2,16-17). De plus, le verset 37 du Psaume 106 (= 105,37 LXx), un autre résumé poétique de l'histoire d'Israël, dépend visiblement de ce passage et nous verrons qu'il y introduit des nouveautés significatives. Enfin, le même motif est repris presque mot à mot

3 Le deuxième discours est constitué par les bénédictions du chapitre 33 .

4 Deut 32,16-17. 
dans le chapitre 4 du livre de Baruch où il est recontextualisé à l'intérieur d'un éloge à la sagesse ${ }^{5}$.

Le dossier des «dieux des autres » est, en outre, complété par deux passages du chapitre 65 du livre d'Isaïe (versets 3 et 11) : même en l'absence d'une relation de dépendance littéraire directe à l'égard de Deut 32, ces deux versets contiennent des références polémiques aux dieux étrangers pour lesquels on brûle de l'encens et on prépare une table, et qui sont encore appelés « démons » dans le texte grec. Mais avant d'analyser la fonction des démons dans les textes grecs qui composent ce corpus, il faut commencer par clarifier le statut des šédîm dans le Chant de Moïse.

\section{1 \\ Formes et représentations du divin en Deutéronome $3^{2}$}

La compréhension de la référence aux démons en Deut 32,17 ne peut pas être séparée de la contextualisation historique plus générale du chant, qui est de ce point de vue l'un des textes les plus débattus de toute la Bible hébraïque. Le problème a deux volets. Un premier volet concerne la datation du texte considéré par la grande majorité des chercheurs comme une unité littéraire au moins pour les versets $1-43$. En effet, dès le XIX ${ }^{\mathrm{e}}$ siècle jusqu'à récemment, le spectre des datations proposées s'étend de la fin du deuxième millénaire avant notre ère au début de l'époque hellénistique et comprend plusieurs solutions intermédiaires ${ }^{6}$. L'incertitude est due, d'un côté, à l'absence de références historiques précises à l'intérieur du chant, qui présente néanmoins une série de parallèles avec la littérature prophétique, et, de l'autre côté, à la difficulté de catégorisation du poème à l'intérieur d'un genre littéraire défini. Les arguments pour une datation haute s'appuient davantage sur l'évaluation des références intertextuelles ainsi que sur des remarques de nature linguistique: deux critères qui sont problématiques du point de vue méthodologique ${ }^{7}$. En revanche, plusieurs chercheurs ont observé des parallèles importants avec

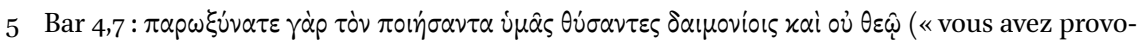
qué celui qui vous a créés en sacrifiant à des démons et non à Dieu »).

6 Une datation au XI ${ }^{\mathrm{e}}$ siècle av. n. è. a été proposée, entre autres, par Cassuto 1973 (première publication 1938); Eissfeldt 1958; Albright 1959; Mendenhall 1975; Moor 1990 ; Tigay 1996, p. 511-512. E. Wright 1962 défend l'idée d'une datation plus tardive, mais néanmoins préexilique; Tournay 196o et Carrillo Alday 1970 situent la composition pendant l'exil. Pour une datation à l'époque postexilique, voir par exemple Meyer 1961; M. Smith 2010 ; E. Otto 2017. Pour un état de la question détaillé et accompagné d'une bibliographie complète, voir récemment E. Otto 2017, p. 2157-2162 (et p. 2131-2143 pour les références bibliographiques).

7 Entre autres, Albright 1959; Kim 2004; Bergey 2003; Gile 2011; voir également Thiessen 2004 ; Leuchter 2007. Pour l'impossibilité d'une évaluation chronologique basée sur des éléments linguistiques, voir l'analyse de Sanders 1996. 
la littérature prophétique plus tardive (notamment le deuxième et troisième Isaie), avec les textes sapientiaux ainsi qu'avec des textes apocalyptiques. Une datation du texte à l'époque postexilique, au moins dans sa forme finale, est donc à considérer comme plus probable, notamment au vu de la fonction de ce chapitre comme charnière entre la Torah et le reste des traditions littéraires qui composent la Bible hébraïque ${ }^{8}$.

Le deuxième volet, qui demeure également très discuté, est celui de l'origine du texte, qui semble différer de celle du reste du Deutéronome. D'après plusieurs auteurs, nous aurions ici affaire à un poème, à l'origine, indépendant qui aurait, ensuite, été ajouté au rouleau du Deutéronome ${ }^{9}$. À cet égard, il faut remarquer que même les chercheurs qui défendent une datation relativement tardive de Deut 32 estiment souvent qu'il contient des traces d'une mythologie très ancienne, interprétée comme le «reste » d'un arrière-plan polythéiste ${ }^{10}$. Parmi les composants d'un tel arrière-plan est souvent indiquée la mention de Deber et Resheph aux versets 22-24a, que j'ai déjà discuté au chapitre 4. Le mythe de la répartition du monde et de ses habitants opérée par le dieu 'Elyôn qui est évoqué aux versets 8-9 est d'habitude considéré comme faisant partie de cet héritage mythologique ancien: nous reviendrons sur ce point. L'hypothèse d'une origine séparée du Chant de Moïse a, cependant, été mise en question par d'autres études récentes qui défendent l'idée que le chapitre 32 aurait plutôt été composé expressément pour son contexte ${ }^{11}$. En outre, le fait que le texte circulait à Qumrân sur un rouleau séparé, comme l'atteste 4QDeut ${ }^{912}$, ne doit pas nécessairement être un argument probant pour une origine indépendante. L'usage liturgique du poème, confirmé par plusieurs passages rabbiniques, peut bien correspondre à un développement secondaire des fonctions du texte ; il faut, en outre, observer que des versets du chapitre 32 étaient également recopiés sous d'autres formes à Qumrân, par exemple combinés avec des extraits d'autres chapitres du Deutéronome (4QDeutj $)^{13}$ ou d'autres livres du Pentateuque (4QDeut $\left.{ }^{\mathrm{k}}\right)^{14}$. N'étant pas en mesure de résoudre le problème, qui mériterait une discussion séparée, je me contenterai de deux remarques générales à propos des rapports entre le Chant de Moïse et le reste du Deutéronome. La première concerne le contenu: en dépit de toutes

8 Voir, à cet égard, l'analyse convaincante de E. Otto 20o9; id. 2017, p. 2164-2173. Pour une datation à l'époque perse, voir également Meyer 1961.

$9 \quad$ Par exemple Eissfeldt 1958; Tigay 1996 ; Sanders 1996.

10 Voir, notamment, M. Weippert 1990 ; M. Smith 2010, p. 195-212.

11 Gosse 1995; E. Otto 2009, 2017.

$12=4 Q_{44}$ (Skehan et Ulrich dans Ulrich et al. 1995, DJD 14, p. 137-142), voir p. 138.

$13=4$ Q37 (Duncan dans Ulrich et al. 1995, DJD 14, p. 75-92), contenait probablement Deut $32,1-9$.

$14=4$ Q38 (Duncan dans Ulrich et al. 1995, DJD 14, p. 93-98), contient Deut 32,17-18. 
ses spécificités, le discours mené par Moïse en Deut 32 reste centré sur deux thèmes qui sont pleinement et fondamentalement «deutéronomiques», à savoir celui de l'élection d'Israël par Yhwh et celui du rapport de Yhwh avec les autres dieux. La deuxième remarque est d'ordre méthodologique et invite à exercer la plus grande prudence envers tout paradigme qui appelle en cause des «survivances ». Comme je l'ai déjà souligné dans l'introduction, ces modèles présupposent généralement une opposition rigide et historiquement non vérifiée entre polythéisme et monothéisme, menant par conséquent à un mauvais usage de ces deux catégories religieuses qui - il faut le rappeler - sont modernes et non anciennes ${ }^{15}$.

À ce propos, je me propose d'aborder la question des éléments mythologiques du poème par une approche différente qui n'est pas focalisée sur l'opposition entre monothéisme et polythéisme. En mettant l'accent sur le rôle attribué aux démons dans ce chant, je me concentrerai davantage sur deux aspects qui, jusqu'à présent et la plupart du temps, ont été traités séparément. Dans les pages qui suivent, j'essayerai de mettre en relation la vénération des šédîm, dont le culte est condamné aux versets 16-17, avec le mythe de l'élection d'Israël, qui est thématisée aux versets $8-9$ ainsi qu'à la fin du poème au verset 43. L'étude de ces passages soulève deux questions fondamentales. La première concerne la relation entre le dieu 'Elyôn et le dieu Yhwh dans les versets 8-9. D'après plusieurs chercheurs, ces versets attesteraient un état ancien de la religion d'Israël où 'Elyôn, littéralement «le très-haut», ne serait pas encore devenu une épithète de Yhwh, comme dans la plupart de la Bible hébraïque, mais serait à considérer comme le nom d'une divinité distincte. Une telle distinction soulève néanmoins le problème du rapport entre cette représentation et celle du verset 43 où Yhwh apparaît comme la divinité suprême qui gouverne l'univers. La deuxième question concerne le rapport entre Yhwh, sa cour céleste des « fils divins » représentée dans les versets 8-9 et 43, et les puissances mentionnées aux versets 16-21, qualifiées, comme nous venons de le voir, comme des «démons» et des nouveaux dieux. Comment peut-on interpréter ces deux représentations de manière cohérente et intégrée ? Et, lorsqu'on arrive à articuler entre eux ces deux scénarios divins, quelles sont les conclusions à en tirer pour l'interprétation plus générale de Deut $3^{2}$ ?

\subsection{Yhwh, 'Elyôn et l'élection d'Israël}

Pour répondre à ces questions il faut d'abord rigoureusement examiner nos textes, notamment en faisant un détour sur le mythe des origines qui est raconté par Moïse au début du poème. Moïse commence son discours en invoquant le ciel et la terre comme témoins de ses dernières paroles, qui descendent du 
ciel et sont destinées à servir d'enseignement universel (versets 1-3). Ensuite, il exhorte les Israélites à se souvenir de leur passé à partir du début de leurs origines (versets 6-7). La narration des origines d'Israël ne correspond pas au récit de l'Exode, comme l'on pourrait s'y attendre. En revanche, elle a lieu dans la cour céleste (versets 8-9) au moment où la divinité décide de partager le genre humain selon le nombre des êtres divins et choisit de garder Israël sous son contrôle direct. Pour la reconstruction du texte originel du verset 8 , les témoignages de la LXx et des manuscrits de Qumrân sont fondamentaux ${ }^{16}$, comme le montre le tableau 4 :

TABLEAU 4 Deutéronome 32,8

\begin{tabular}{|c|c|c|}
\hline TM & ${ }_{4 Q D e u t}{ }^{j}$ & LXX \\
\hline 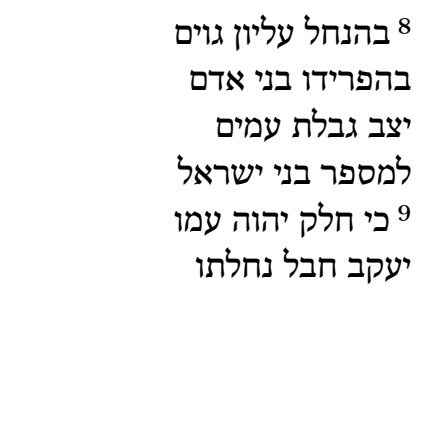 & 8 בני אלוהים & 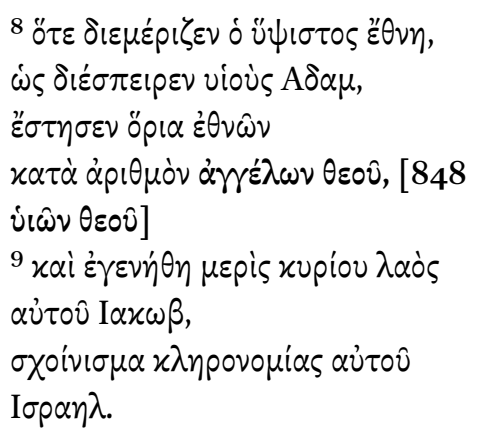 \\
\hline $\begin{array}{l}{ }^{8} \text { Quand le Très-Haut a } \\
\text { donné en héritage les } \\
\text { peuples, } \\
\text { Quand il a séparé les êtres } \\
\text { humains (litt. «les fils } \\
\text { d'Adam ») } \\
\text { Il a établi les limites des } \\
\text { peuples selon le nombre } \\
\text { des fils d'Israël, } \\
{ }^{9} \text { Vraiment la portion de } \\
\text { Yhwh est son peuple, } \\
\text { Jacob son héritage. }\end{array}$ & $\begin{array}{l}8 \text { les fils } \\
\text { divins }\end{array}$ & $\begin{array}{l}{ }^{8} \text { Quand le très haut a partagé les } \\
\text { peuples, lorsqu'il a distribué les } \\
\text { fils d'Adam } \\
\text { il a établi les limites des peuples } \\
\text { selon le nombre des anges des } \\
\text { dieux/des fils de dieu, } \\
{ }^{9} \text { et la part du Seigneur fut son } \\
\text { peuple Jacob, } \\
\text { La portion de son héritage, Israël. }\end{array}$ \\
\hline
\end{tabular}

16 Pour le texte grec l'édition de référence est celle de Wevers 1977 ; les fragments de Qumrân sont cités d'après l'édition de Julie Duncan (dans Ulrich et al. 1995, DJD 14, col. XII, l. 14, p. 9o). 
Alors que nous lisons, dans le $\mathrm{TM}^{17}$, que les bənê 'ādām, les « fils d'Adam », sont distribués selon le nombre des bənêyiśrāèel, « des fils d'Israël», les manus-

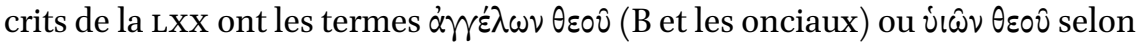
le Papyrus Fouad ${ }^{18}$. Deux manuscrits qumrâniens utilisent bny 'l (suivi par un espace où nous n'arrivons plus à reconstruire le texte) et bry 'lwhym et confirment ainsi que la traduction grecque reflète la version originale de l'hébreu ${ }^{19}$. Banê élîm ou banê élōhîm sont deux expressions équivalentes pour désigner les membres de la cour céleste dans plusieurs passages bibliques de la Genèse, des Psaumes et du livre de Job ${ }^{20}$. Dans tous ces passages, la LXX lit vioi $\theta \varepsilon \circ \hat{\imath}$

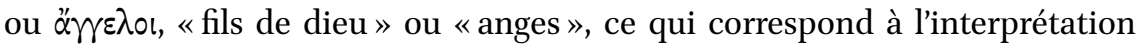
usuelle des banê élōhîm de la part des traducteurs grecs ${ }^{21}$. Comme il a d'ailleurs été reconnu depuis longtemps, la version originale de l'hébreu devait donc correspondre à l'une des deux expressions attestées à Qumrân et dans le Papyrus Fouad, alors que la version du TM est le résultat d'une correction tardive, motivée par des soucis d'ordre théologique ${ }^{22}$ sur lesquels je reviendrai.

La relation entre les versets 8 et 9 est, en revanche, moins claire. D'après les chercheurs qui voient dans ce passage deux divinités distinctes ${ }^{23}$, 'Elyôn serait le nom du chef du panthéon (ou une épithète du dieu El en tant que chef du panthéon) en charge du partage de l'univers parmi les autres agents divins. Cette situation reflèterait donc un stade de la religion d'Israël où Yhwh et $\mathrm{El} /$ 'Elyôn n'auraient pas encore été identifiés l'un à l'autre. Parmi les puissances divines Yhwh émergerait donc comme celui qui reçoit en sort Israël: ceci correspondrait aux «restes» supposés d'une ancienne structure polythéiste dominé par le dieu El où Yhwh ne jouerait qu'un rôle subordonné. Ce serait ici une attestation assez exceptionnelle étant donné que, dans la grande majorité des cas dans la Bible hébraïque, 'Elyôn est utilisé comme épithète de Yhwh, ou de El lorsque ce dernier est identifié à Yhwh ${ }^{24}$, ou est utilisé dans des contextes

17 Le Pentateuque samaritain, les Targums et la Vulgate suivent la version du тм.

18 No. 848 dans l'édition de Wevers 1977. Cette version se trouve également dans une minuscule $\left(106^{c}\right)$ ainsi que dans le mss 85 du texte d'Aquila (Field 1964, p. 320, note 12): elle est préférée par Wevers (contra Rahlfs qui choisit ä $\gamma \varepsilon \lambda_{01}$ ).

19 Skehan 1954; Ulrich (dans Ulrich et al. 1995, DJD 14, fr. 34, p. 80-90).

$20 \quad$ Par exemple en Gen 6,2 et 4; Ps 82, Job 1-2 et Job 38.

21 Voir Dogniez dans Dogniez et Scopello 2006, p. 180-183, et infra, § 8.1.1.

22 Voir Barthélemy 1978, p. 101-110; Himbaza 2002.

23 Voir, entre autres, Zobel 2001 ; Weippert 1990 ; Sanders 1996; Wyatt 2007 ; White 2014.

24 Pour l'identification entre Yhwh et El à un stade ancien de la religion d'Israël, voir M. Smith 2001, p. 135-145. Sur la possibilité d'un culte rendu à Yhwh comme El 'Elyôn à Shilo à l'époque monarchique, voir Seow 1989, p. 41-54. 
où la référence à Yhwh est évidente même en l'absence du tétragramme ${ }^{25}$. Or, cette interprétation, qui a connu un large succès, se heurte à quelques difficultés syntaxiques et, comme l'a bien montré Konrad Schmid ${ }^{26}$, elle n'est pas non plus la plus convaincante qui soit. En effet, une lecture attentive du passage suggère que Yhwh et 'Elyôn sont à identifier comme une seule divinité et que 'Elyôn doit être compris comme une épithète de Yhwh. Alors que le verset 8 décrit l'action divine en adoptant une perspective universaliste en qualifiant la divinité comme le dirigeant «suprême » ('elyôn) de l'humanité (gôyim), la mention du nom de Yhwh au verset 9 souligne son caractère de dieu «national » en mettant l'accent sur sa relation étroite avec Israël et son histoire (Jacob). La conjonction $k \hat{\imath}$, qui relie les deux versets, a ici une fonction assévérative, à savoir qu'elle renforce l'affirmation du lien entre Yhwh et son peuple. Cela a du sens du point de vue syntaxique seulement si l'on comprend Yhwh et 'Elyôn comme deux noms d'une seule puissance divine, qui désignent deux attributs ou champs d'actions différents de la même divinité. D'autres observations viennent renforcer la proposition de Schmid. La présence du terme hẹeleq au verset 9, qui désigne Israël comme la « portion» de Yhwh, renforce la réciprocité du lien entre le peuple et sa divinité patronne, lien qui est considéré comme remontant au début de l'histoire universelle. La même racine apparaît en Deut 4,19-20 et 29,25 (26) où Yhwh met en garde son peuple contre la servitude envers d'autres dieux qu'il n'a pas «attribués » (hâlaq) à Israël mais à d'autres peuples, comme nous le lisons, par exemple, en Deut 4,19-20:

25 Voir, par exemple, Ps 9,3 et 107,11. Voir, sur ce sujet, Niehr 1990. Dans quelques passages l'identification entre El 'Elyôn et Yhwh est douteuse, notamment en Gen 14,18-22 et Num 24,16. En Gen 14, Melchisédech est décrit comme prêtre de El 'Elyôn et comme roi de Salem et bénit Abraham par le nom de El 'Elyôn : dans ce cas l'insertion du nom Yhwh au verset 22 est d'habitude considérée comme une glose explicative. En Num 24,16, il est question du devin Balaam qui est présenté comme «celui qui écoute les paroles de $\mathrm{El}$, qui possède la connaissance d"Elyôn et qui voit les visions de Shadday ». Ce passage a été longtemps considéré comme l'une des références les plus anciennes au dieu 'Elyôn. Toutefois, la recherche récente a remis en question ces deux consensus, en démontrant que dans les deux cas nous avons affaire à des compositions tardives. La référence à Melchisédech pourrait être une insertion secondaire en Gen 14 (Pour une origine tardive de la narration concernant Melchisédech, voir, par exemple, Granerød 2010, en part. p. 153-171). L'oracle de Balaam (Num 24,15-24) est, pour un nombre croissant de chercheurs, un texte remanié à plusieurs reprises et probablement ajouté au chapitre 24 à un stade où l'association entre El et Yhwh était désormais bien établie.

26 Schmid 2006. Une interprétation similaire avait déjà été proposée par Carrillo Alday 1970, p. 53-57, et Schenker 1997. Voir également M. Smith 2010, p. 203 sqq. 


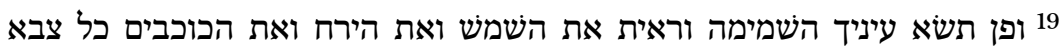

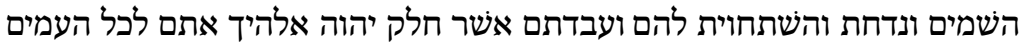
תחת כל השמים 20 ואתכם לקח יהוה ויוצא אתכם מכור הברזל ממצרים להיות לו לעם נחלה כיום הזה

${ }^{19} \mathrm{Ne}$ lève pas tes yeux vers le ciel, (ne) regarde pas le soleil, la lune et les étoiles, toute l'armée des cieux, et ne te laisse pas entraîner à te prosterner devant eux et à les servir. Car ils sont la part que Yhwh ton Dieu a donnée à tous les peuples qui sont partout sous le ciel.

${ }^{20}$ Mais vous, Yhwh vous a pris et il vous a fait sortir de cette fournaise à fondre le fer, de l'Égypte, pour que vous deveniez son peuple, son patrimoine, comme vous l'êtes aujourd'hui.

Il paraît, d'ailleurs, manifeste que le traducteur grec a perçu et gardé cette dialectique entre une perspective universelle et une perspective plus locale ou nationale : au verset 8 , il utilise le verbe $\delta$ ı $\mu \varepsilon p i \zeta \omega$, « séparer », «faire les portions », pour traduire le Hiphil de $h h l$, , « donner en sorte », normalement tra-

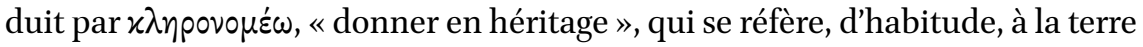
d'Israël. En outre, il ajoute le nom «Israël» au verset $9 \mathrm{~b}$ pour rendre explicite l'équivalence entre Jacob et Israël, à l'instar d'autres cas similaires dans la $\mathrm{LXX}^{27}$.

L'hypothèse qu'en Deut 32,8 'Elyôn soit à considérer comme une épithète de Yhwh peut également être renforcée par le contexte plus large de ce passage qui comprend les versets 6-7. Ici Yhwh est présenté comme «père » ('ābîka $)$, celui qui a établi $(k w n)$ et créé $(q n h)$ : il a donc des traits qui le rapprochent de la fonction traditionnellement attribuée au dieu El dans les panthéons ouest-sémitiques. Mark Smith a souligné les parallèles étroits de vocabulaire entre ces versets et les descriptions du dieu El dans les textes ougaritiques ${ }^{28}$. Or, si Yhwh est déjà présenté comme un dieu de type El aux versets 6-7, il serait néanmoins surprenant qu'il perde ces traits dans les versets suivant immédiatement, qui sont logiquement liés à ce qui précède.

En outre, l'idée d'une distinction entre 'Elyôn et Yhwh aux versets 8-9 soulève un problème de cohérence par rapport à la fin du poème, au verset 43, où Yhwh est clairement reconnu comme le chef incontesté du panthéon car tous

27 Mepic, «part» apparaît également au verset 9 pour traduire hẹeleq ; voir, sur ces aspects, Dogniez dans Dogniez et Harl 1992, p. 325-326.

28 Smith 2002, p. 32-40, qui cite $K T U 1.3$ V 35-36; 1.4 I 4-5; + KAI 129, l. 1. Pour l'association étroite de la racine $q n h$ avec El en sémitique, voir également Seow 1989, p. 19-22. 
les autres dieux sont invités à se réjouir avec lui. Là encore, il faut tenir compte de la pluralité textuelle attestée par le TM, la LXX et les manuscrits de la Mer Morte, résumée dans le tableau suivant (tableau 5):

tableau 5 Deutéronome 32,43

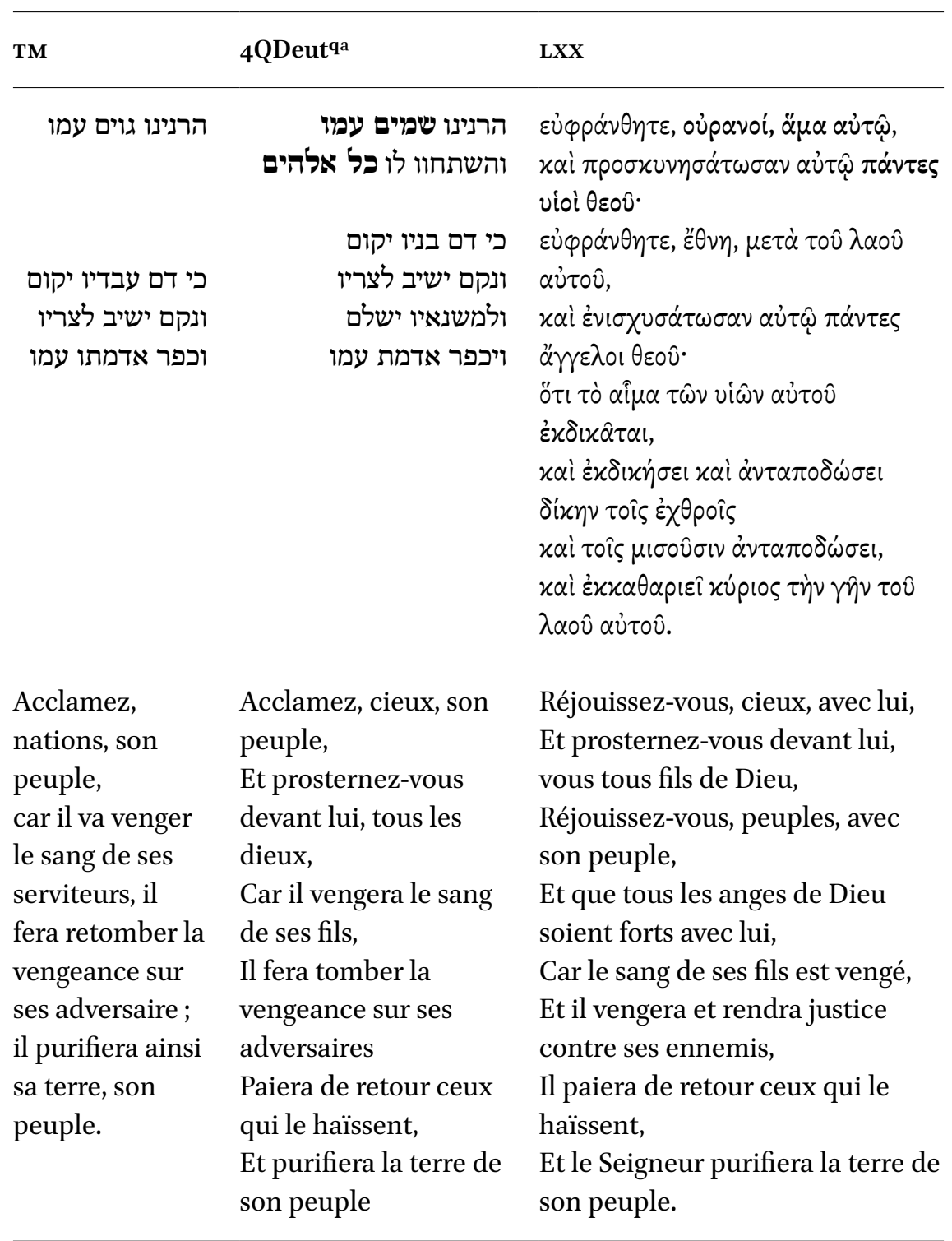

a Col. II, fr. 5 ii (Ulrich et al. 1995, DJD 14, p. 141-142). 
La version du TM est la plus courte, la référence aux cieux et à la cour céleste en a disparu, et l'invitation est adressée aux nations (gôyìm) afin qu'elles se réjouissent avec le peuple d'Israël ('ammô). Les problèmes de critique textuelle concernant ce verset ont été examinés en détail, entre autres, par Maurice Bogaert et par Arje van der Kooij29. Alors qu'il y a un consensus sur le fait que la version la plus longue, à savoir celle de huit côla attestée par la LXX, ne peut pas refléter la forme originelle du texte, il apparait moins clair de savoir si cette dernière aurait consisté en quatre côla (comme dans le TM), ou en 6 (comme en 4 Q Deut ${ }^{\mathrm{q}}$ ); il est également difficile de savoir à quel stade de la transmission du texte les changements ont été introduits. La version pré-massorétique originelle ne peut vraisemblablement plus être reconstruite en détail. Toutefois, nombre de raisons structurelles suggèrent que celle-ci était de six ou de quatre $c o ̂ l a^{30}$ et qu'elle contenait encore dans les deux premiers côla une référence aux cieux (šāmayim) et aux êtres divins ('ělōhîm ou bənê 'ělōhîm), présents à la fois dans la LXX et à Qumrân. Par conséquent, la forme massorétique du premier côlon du verset 43 paraît être une correction allant dans la même direction que celle du verset 8 : les deux ont pour résultat la disparition de toute référence à la cour céleste et soulignent, en revanche, la relation entre Yhwh et Israël ${ }^{31}$. Lorsque l'on considère la forme pré-massorétique du verset 43, on remarque néanmoins qu'elle contenait un renvoi au début du chant car elle s'adresse aux cieux comme dans le verset 1 . De plus, elle est articulée de manière cohérente avec le verset 8 . La représentation des versets 8 et 43 où les populations sont distribuées sur la terre et assignées chacune à la tutelle d'une puissance divine trouve un parallèle relativement proche dans la «table des nations » décrite en Gen 10 où la diffusion des fils de Noé est à l'origine des différentes nations du monde. En dehors de Deut 32,8, l'association entre le verbe « séparer» ( $p r d)$ et les «nations » (gôyìm) apparaît seulement en Gen 10,5 et $32^{32}$. En revanche, le parallèle ougaritique qui est parfois évoqué pour démontrer la nature «archaïque » du mythe de Deut 32,8-9, à savoir la mention des soixante-dix fils de El et Athirat, n'a pas de véritable correspondant dans ce

29 Bogaert 1985; Van der Kooij 1994; voir également Barthélemy 1978, p. 101-110; Himbaza 2002.

30 L'idée d'une version pré-massorétique à six côla est la position dominante dans la recherche actuelle : pour une mise au point, voir MacDonald 2012, p. 92-93.

31 Comme cela a déjà été observé, la correction massorétique aux versets 8 et 43 vise à créer un lien avec les soixante-dix fils de Jacob nommés en Gen 46,27 et Ex 1,5, dont le nombre a été corrigé de soixante-quinze au chiffre symbolique de soixante-dix (voir Himbaza 2002, p. 540).

32 Gen 10,5: «à partir d'eux les régions des peuples furent réparties »; 10,32 : « c'est à partir d'eux que les peuples furent répartis sur la terre». 
passage car, nulle part, il n'est explicitement fait mention dans les mythes ougaritiques d'une répartition du territoire parmi les fils du couple divin ${ }^{33}$. En outre, l'expression bənê êlōhîm dans la Bible hébraïque ne doit pas nécessairement être comprise au sens généalogique car elle peut simplement indiquer les membres d'un groupe ou d'une classe, à savoir, ici, celle des divinités admises à la cour céleste.

Par rapport à Gen 10, il faut toutefois préciser que, bien que la table des nations implique l'idée d'un partage du territoire parmi les différents peuples du monde, cette idée ne semble pas soutenue par la même perspective universaliste qui est manifeste en Deut 32,8-9 et 43 : en ce sens, les passages du Chant de Moïse ne disposent pas de véritables parallèles bibliques et ils ne semblent pas avoir de précédent dans la mythologie du Proche Orient ancien. Par contre, les représentations de Gen 10 et de Deut 32 sont combinées dans des traditions du Second Temple, comme, par exemple, dans le livre des Jubilés ${ }^{34}$.Comme l'observe justement Mark Smith ${ }^{35}$, l'idée d'une distribution de « gouverneurs » sur chaque peuple et d'Israël comme «portion » de Yhwh est reprise par un passage de Ben Sira et intégrée dans l'histoire des origines de l'homme : $\dot{x} x \alpha ́ \sigma \tau \omega$

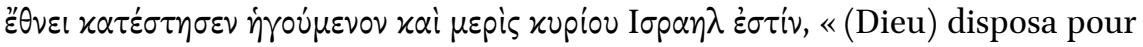
chaque peuple un gouvernant, et Israël est la part du Seigneur ${ }^{36}$. Ces parallèles témoignent de la popularité de ce mythe pendant l'époque hellénistique.

\subsection{Au-dedans et en dehors de la cour céleste}

L'hypothèse d'interprétation qui a été proposée pour les versets 8-9 et 43 nous amène directement à approfondir le statut des dieux étrangers qui sont l'objet de la polémique des versets $16-21$ car ce statut soulève la question de la relation entre deux scénarios qui, à première vue, apparaissent très différents. Du verset 16 au verset 21, le reproche de Moïse s'adresse aux Israélites en raison de l'ingratitude qu'ils ont montrée à l'égard de Yhwh en se tournant vers d'autres divinités. La péricope en hébreu lit comme suit:

16 Ils l'ont rendu jaloux par des cultes étrangers,

Par des abominations ils l'ont provoqué

(yakîsuhû

33 Pace Wyatt 2007.

34 Jub 15,30-32.

35 M. Smith 2010, p. 208-209.

36 Sir 17,17. Dans le contexte de l'Alexandrie hellénistique où se situe la traduction, nous

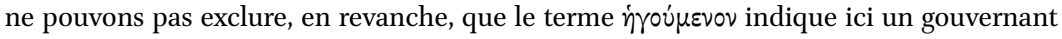
humain et non divin. 

17 17 Ils ont sacrifié aux démons (šēelîm), qui ne sont אלהים לא ידעום pas Dieu (lō'’èlōah),
divinités qu'ils ne connaissaient pas, nouvelles, elles sont arrivées récemment, leurs pères ne les craignaient pas.
${ }^{18}$ Le rocher qui t’a engendré, tu l'as abandonné;
tu as oublié le dieu qui t'a mis au monde.
${ }^{19}$ Yhwh a vu,
il a rejeté ses fils et ses filles car il a été vexé,
20 et il a dit: «je leur cacherai ma face,
et je verrai ce qu'il en est d'eux,
car ils sont une génération perverse,
fils dans lesquels n'y a aucune fiabilité.

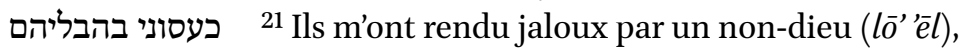
Ils m'ont provoqué par leurs pratiques vaines
בגוי נבל אכעיסם (bahablêhem),

Et moi je les rendrai jaloux par un non-peuple,

par une nation folle je les provoquerai ».

L'idée qu'Israël ne doit pas servir d'autres dieux car ces derniers ont été assignés à d'autres nations qu'Israël n'est pas étrangère au Deutéronome. Nous avons déjà vu la formulation de Deut 4,19-20. Il est possible de citer encore le passage de Deut 29,25, où il est question des autres dieux ('élōhîm 'ăhểrîm). Ce dernier montre des similitudes considérables avec Deut 32,8-9 (l'idée du partage entre dieux et peuples) et 16 (la conviction que les Israélites rendent un culte à des dieux étrangers) ${ }^{37}$ :

\section{וילכו ויעבדו אלהים אחרים וישתחוו להם ידה אלהים אשר לא ידעום ולא חלק להם וים וערות}

Ils sont allés servir d'autres dieux, ils se sont prosternés devant eux, dieux qu'ils ne connaissaient pas, que (Yhwh) ne leur avait pas donnés en partage.

Pour un passage similaire, voir également Deut 17,3. Le thème des « autres dieux » qui ne sont pas les divinités ancestrales est courant dans le Deutéronome: voir, par exemple, Deut 4,27-28 et 28,36 et 64 . Dans ces derniers cas, la référence est vraisemblablement aux images divines babyloniennes (« du bois et de la pierre») qui auraient été vénérées pendant l'Exil, et il n'est pas fait mention du motif du «partage » entre nations et puissances divines. 
Par rapport à ces passages deutéronomiques de même thématique, Deut 32,16-21 se caractérise par une violence polémique considérable. Le statut divin de ces puissances est effectivement nié, ce qui n'arrive ni en Deut 4 ni en Deut 29. Non seulement ils sont définis comme des démons (šêdîm) et comme des êtres non divins (lō' 'ělōah, verset 17a), mais leur vénération constitue une abomination (tốếbot, verset 16); ils sont également condamnés en tant que divinités nouvelles ('élōhîm hăădāšm, verset $17 \mathrm{~b}$ ), arrivées récemment et inconnues des pères, à savoir des divinités non traditionnelles. Le sommet de la polémique se trouve au verset 21 où ils sont définis comme «non-dieux »

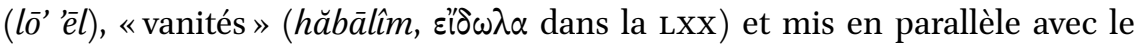
«non-peuple», la nation folle que Yhwh enverra contre Israël comme juste punition pour ses méfaits ${ }^{38}$.

La question centrale ici concerne la manière dont ces puissances, clairement négatives et dénuées de pouvoir, entrent en relation avec les membres de l'assemblée divine auxquels chaque nation a été attribuée et qui sont encore invités à célébrer Yhwh à la fin du chant. Une explication récente proposée par Nathan MacDonald considère que les divinités mentionnées au verset 17 seraient les mêmes membres de la cour céleste qu'aux versets 8-9 et 43, mais considérés sous un angle différent. Autrement dit, ils seraient indignes du titre divin seulement lorsqu'ils sont comparés avec la magnificence de Yhwh ${ }^{39}$. Cette vision quelque peu harmonisante n'explique pourtant pas encore la différence considérable d'emphase qui existe entre les passages du chant qui décrivent la cour céleste et ceux qui sont focalisés sur la polémique contre les démons ou les nouveaux dieux. En outre, le verset 17 insiste sur le fait qu'ils soient arrivés récemment et ne soient pas des divinités traditionnelles. Il me paraît donc difficile qu'il puisse s'agir des mêmes divinités qui étaient présentes au début de l'histoire, lors du partage du monde parmi les êtres divins. Par conséquent, une distinction supplémentaire doit être envisagée entre les bənê ělōhîm des versets 8-9 et les 'élōhîm hăăăšìm des versets 17-21. Cette interprétation nous amène donc à envisager un scénario articulé sur plusieurs niveaux, dans lequel on distingue les banê ělōhîm, puissances compatibles avec la souveraineté de Yhwh et subordonnées à son pouvoir, d'autres divinités allogènes qui

$38 \quad$ Malgré les différentes interprétations proposées, il est impossible d'identifier ce «(non)peuple», et il faut sérieusement considérer l'hypothèse que le manque d'identification soit ici intentionnel, tout comme pour l'expression précédente «non-dieu». Pour quelques hypothèses d'identification du non-peuple, notamment avec les Babyloniens, voir néanmoins Alday 1970, p. 89-92.

39 MacDonald 2012, p. 91-92: «The significance of terms is relative, not absolute. That is, the existence of other nations and other gods is not denied, but rather these 'gods' and 'people' are not worthy of the titles in comparison with Yhwh and Israel ». 
ne peuvent pas être intégrées à la cour divine et qui sont présentées comme des dieux nouveaux, voire comme des démons. Un tel scénario a des implications considérables pour la compréhension du poème dans son ensemble. Lorsqu'on l'analyse à partir de cette perspective, le message central du Chant de Moïse n'est plus focalisé sur l'expression du monothéisme, comme on l'a si souvent soutenu ${ }^{40}$. Le poème porte, en revanche, sur l'articulation des puissances divines dont un groupe traditionnel, celui des «fils divins », est placé sous l'autorité de Yhwh alors que l'autre est représenté par un ensemble de puissances nouvelles et venues de l'extérieur qui échappent au contrôle de la divinité patronne. Nous nous approchons d'une forme de division des pouvoirs qu'on retrouvera dans plusieurs traditions du Second Temple et que je serais tentée de définir comme dualiste ${ }^{41}$.

La présence d'un système de pouvoirs divins aussi complexe dans la version du texte telle qu'elle est préservée à Qumrân et dans la LXX ramène enfin notre attention sur les différences entre ces versions et celle du тм. Ce dernier présente un scénario plus simple, dans la mesure où toute référence à la cour céleste a été intentionnellement évacuée, et le contraste entre Yhwh et les autres dieux est présenté de manière plus standardisée. Là encore, il n'était probablement pas question d'«anciennes traces polythéistes» qui auraient dérangé les scribes, d'autant plus que les fils divins sont bien présents ailleurs dans la Bible hébraïque et que d'autres divinités sont nommées sans accent polémique particulier dans le Deutéronome même, aux chapitres 4 et 29. Le problème devient en revanche plus compréhensible lorsque l'on considère que la vision de la cour céleste en Deut $3^{2}$ est transmise aux Israélites par Moïse. Les deux corrections massorétiques aux versets 8 et 43 visent alors à éliminer de la bouche de Moïse, et ainsi du Pentateuque en général, les références à la cour céleste qui, dans les versions prémassorétiques, encadraient l'intégralité du chant (versets 1, 8-9 et 43). On peut donc envisager que certains scribes aient voulu corriger une image trop « exotérique » de la révélation de Moïse et, par conséquent, de sa loi, notamment en raison de la référence à la cour céleste. Ils ont ainsi restauré un imaginaire de la révélation divine moins riche et moins dynamique, mais en quelque sorte plus «classique» et, de ce fait, peut-être plus acceptable à leurs yeux.

40 Je suis d'accord sur ce point avec les conclusions formulées par MacDonald 2012 à propos de Deut 32,39.

41 Ces remarques ont également une implication pour la datation de Deut 32, dans la mesure où elles appuient une datation tardive du poème, surtout si nous les combinons avec la présence du jugement final où la vanité des autres divinités sera manifestée de manière complète (versets 37-38). On est proche d'une conception apocalyptique de l'histoire et de la révélation, ce qui est par ailleurs en tension avec d'autres passages du Deutéronome. 


\subsection{Les šêdîm, Shadday et le dieu Shed: une mise au point}

Avant de nous tourner vers le texte de la LXx pour évaluer la manière dont est développée la vision des dieux étrangers comme démons, il faut revenir sur la question de l'étymologie des šêdîm brièvement présentée au chapitre 3 . La majorité des dictionnaires considère ce mot comme un dérivé de l'accadien šedu, «esprit ${ }^{42}$, qui peut indiquer à la fois un esprit protecteur et une entité malfaisante et redoutable, surtout dans ses occurrences à la forme plurielle. Le terme est attesté en hébreu et dans d'autres langues ouest-sémitiques (judéo-araméen, syriaque et mandaïque), surtout à partir de la période du Second Temple, en différentes formes graphiques (šyd, šyd', šydh et šydt'), mais toujours avec la signification de « démon ». Cette dérivation a parfois été questionnée sur le plan étymologique car certains chercheurs ont préféré mettre en relation les šedîm avec les dieux de type «Shed», attestés au Levant et en Égypte ${ }^{43}$, ainsi qu'avec le titre divin Shadday qui est une des épithètes de Yhwh dans la Bible hébraïque, encore aujourd'hui dépourvu d'une étymologie satisfaisante ${ }^{44}$. Quelques remarques sont donc nécessaires à ce sujet.

Une divinité nommée Shed est connue à Ougarit, bien qu'elle le soit par deux ou trois attestations seulement : son nom est probablement lié à la racine $\check{s} d$ qui indique les champs ouverts et les steppes ${ }^{45}$. À cet égard, il faut remarquer que $\check{s} d y$ pourrait bien être la forme longue de $\check{s} d$, souvent utilisée pour les dieux ougaritains à côté de la forme courte. La présence de Shed en Phénicie est, en revanche, plus douteuse ${ }^{46}$. Une origine égyptienne du Shed sémitique

42 Zimmern 1917, p. 69; Gesenius 1916, s.v., HALOT, s.v.; plus récemment Mankowski 2000, p. 138. Pour le šedu mésopotamien, voir Von Soden (AHw), p. 1208 ; CAD 17/2, s.v.

43 Notamment par Caquot 1952, qui reprend une ancienne hypothèse de Baudissin 1876, p. 130-136 ; ensuite Hackett 1984 ; Delcor 1989. Voir également Xella 1983.

44 La bibliographie sur Shadday est vaste. Pour une synthèse, on peut voir, entre autres, Niehr et Steins 1983 ; Weippert 1997 ; Knauf 1999, p. 749-753; plus récemment Witte 2011. Pour une étude des usages de Shadday dans le livre de Job, voir l'étude classique de Koch 1976. Une dernière proposition étymologique a été avancée par Wilson-Wright 2019. De manière générale, le débat sur l'étymologie de Shadday et les différents résultats auxquels la recherche a abouti ne se sont pas avérés très utiles à la compréhension du fonctionnement du dieu qui est, pour ainsi dire, «derrière » l'épithète. Il faudrait à cet égard garder à l'esprit l'avertissement de James Barr quant au fait que l'étymologie d'un mot nous renseigne beaucoup plus sur son histoire que sur sa véritable signification (Barr 1961, p. 109).

$45 K T U$ 1.108. 12 (= RS 24.252) : 'l šd, « dieu Shed»; KTU 1.179. 8-12 (= RS 92.2016, 11 édité par Caquot et Dalix 20o1): $y d d$ wšd, «le bien aimé et Shed »; KTU 1.166. 13 (= HANI 77/78 A): $\check{s} d q d \check{s}$, «Shed saint ». Contra l'identification de Shed dans ces passages, voir Lipiński 1995, p. $251-253$.

46 L'expression gršd («client de Shed ») sur un sceau provenant de Tyr (Paris, Bibliothèque Nationale, Collection De Clerq, no. 2756, Bordreuil 1986, p. 36) ainsi qu'une inscription 
est également improbable ${ }^{47}$; par contre, le dieu Shed, bien attesté en Égypte depuis le Nouveau Règne comme divinité guérisseuse, est souvent représenté par une iconographie qui a des traits proche-orientaux ${ }^{48}$.

Une forme plurielle šdyn est, en outre, attestée au Levant par l'inscription araméenne de Deir 'Alla, qui remonte à la fin du IX ${ }^{\mathrm{e}}$ siècle ou au début du VIII ${ }^{\mathrm{e}}$ au plus tard. Ici un groupe de divinités dites šdyn intervient au conseil divin et révèle au voyant Balaam des présages néfastes qui vont bientôt s'abattre sur l'humanité. Les šdyn sont mis en parallèle avec les 'lhn (= hébreu 'ělōhîm $)^{49}$ :

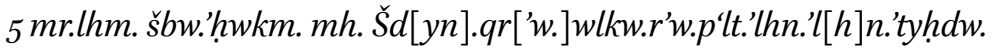
6 wnșbw. Šdyn.mw'd.w'mrw.lš[mš].

Asseyez-vous ! Je vous dirai ce que les Šaddayîn ont juré. Et allez, regardez les gestes des dieux! Les dieux se sont réunis, et les Šaddayîn ont constitué une assemblée et ont dit à Š[amaš]...

Bien que le très mauvais état de conservation de l'inscription ne permette pas de clarifier la relation entre ces deux groupes de divinités, il semble assuré que les šdyn sont des membres de l'assemblée céleste qui invitent une autre

dans le temple d'Eshmun à Sidon qui aurait la forme šdqď̌ («Shed saint») sont de lecture douteuse : voir la critique de Lipiński 1995, p. 254 sqq. La lecture gršd est, toutefois, acceptée par Gubel 1993, p. 118, fig. 41, et par Avigad et Sass 1997, p. 274, no. 736. La formule gr suivie d'un théonyme est attestée dans d'autres noms composés, tels que Gerashtart et Ger'eshmun : voir Avigad et Sass, 1994, p. 274, no. 733-735; Gubel 1993, p. 118, fig. 42 ; DNWSI 1, p. 232, s.v. $g r_{1}$. Mais même si on accepte cette lecture, il s'agit en tout cas d'un témoignage isolé.

47 Cette hypothèse a été soutenue récemment par Valérie Matoïan (2015, surtout p. 265268). Un examen complet du dossier sémitique et égyptien a été proposé par Giuseppina Lenzo et Christophe Nihan lors de leur communication « Shed in Egypt and West-Semitic Deities : A Reexamination», pendant le SBL Annual Meeting, Denver, 18 November 2018. L'analyse détaillée de Lenzo et Nihan ne soutient pas la thèse de Matoïan. En revanche, une influence égyptienne est reconnaissable sur le théonyme phénicien Shadrapha, «Shed est guérisseur », attesté depuis le v siècle et pendant la période hellénistique sur des inscriptions phéniciennes, puniques et araméennes.

48 Bonnet 1971, p. 676-677; Meeks 1971, p. 56-57; Quaegebeur 1984, p. 138-139; pour une dernière mise au point, voir Lenzo, à paraître.

49 Editio princeps Hoftijzer et Van der Kooij 1976, p. 173-174, et p. 275-276. Pour le commentaire, voir également Caquot et Lemaire 1977; Hackett 1984; Weippert et Weippert 1982, p. 88-92 ; Weippert 1991, p. 151-184; Lipiński 1994, p. 103-141 (en part. p. 122-125); Levine 200o, p. 140-145 ; Fales et Grassi 2016, p. 151-158. Je suis la reconstruction du texte proposée par Fales et Grassi, dont je reprends et adapte la traduction. 
divinité (possiblement le dieu solaire $\check{S}^{2} a m s^{50}$ ) à répandre sur la terre obscurité et nuages. Ils apparaissent vraisemblablement comme des puissances liées à la sphère astrale. Certains chercheurs ont reconnu dans ce passage un parallélisme avec les šédîm et les élōhîm qui apparaissent en Deut 32,17. Ils ont donc émis deux hypothèses. La première considère que l'état le plus ancien du texte de Deut 32,17 avait également une forme šdyn (à prononcer šaddayîn) ou bien une forme šdym où le yod faisait partie de la racine; forme qui aurait ensuite été corrigée et ramenée à šêdîm - « démons » - pour éviter le risque d'un rapprochement entre Yhwh et des divinités étrangères. La deuxième hypothèse envisage un lien entre les šdyn et l'épithète Shadday ${ }^{51}$. Or, la possibilité d'une relation entre le Shadday biblique et les Shaddayin de Deir 'Alla ne peut pas être complètement écartée, notamment car, dans la Bible hébraïque, le titre Shadday est souvent accordé à Yhwh en tant que divinité des origines et d'un passé ancien ${ }^{52}$. En outre, ce titre apparaît deux fois dans le livre des Nombres dans la péricope qui a pour protagoniste Balaam ${ }^{53}$. De plus, la présence d'une double forme du théonyme šd/šdy déjà à Ougarit laisse ouverte la question du rapport entre Shadday et les dieux «Shed» au Levant. La permanence d'un nom divin si ancien dans les textes bibliques, vraisemblablement bien postérieurs au $\mathrm{IX}^{\mathrm{e}}$ siècle, n'est pas si surprenante si l'on considère que le théonyme Shed est encore attesté au $\mathrm{I}^{\mathrm{er}}$ siècle de notre ère à Palmyre ${ }^{54}$. Le théonyme a clairement une longue histoire, bien que ses contours soient mal définis, et il est possible que l'épithète biblique Shadday ait, à un moment donné, fait partie de cette histoire.

En revanche, plusieurs éléments invitent à repousser l'inclusion des šêdîm dans ce dossier. Tout d'abord, les domaines d'action de ces puissances sont très différents : les dieux de Deir 'Alla ainsi que le Shed ougaritique apparaissent comme des divinités astrales, liées aux étoiles et aux changements météorologiques; le Shed égyptien est, pour sa part, une divinité guérisseuse, tandis que les šêdîm bibliques sont caractérisés comme des puissances démoniaques exclues de la cour céleste qui ne semblent pas être associées à un champ d'action particulier. En outre, le parallélisme entre 'ělōhîm et šêdîm n'est pas du

$5^{\circ} \quad$ Cette lecture est acceptée par tous les éditeurs à l'exception de Hoftijzer et Van der Kooij (1976) et de Levine (2000) qui y voient la déesse Šagar.

51 Voir Delcor 1989, et également Hackett 1984, p. 85-89; Sanders 1996, p. 182-183, suivis par Stavrakopoulou 2004, p. 272-274. Contra Weippert 1991, 179, note 69. Sur la deuxième hypothèse, voir également Witte 2011, p. 224-225.

$5^{2} \quad$ Notamment la forme El Shadday en Gen 17,$1 ; 28,3 ; 35,11 ; 43,14 ; 48,3 ; 49,25 ;$ Ex 6,3 .

53 Num 24,4 et 16.

54 Son nom apparait dans une inscription du temple de Bel : DNWSI 2, 1111; Cantineau 1931, p. 130-131. 
même ordre que celui entre les 'lhn et les šdyn de l'inscription de Deir 'Alla car en Deut 32,17 l'expression « dieux » ('êlōhîm) est modifiée par la forme qui précède, «non dieu » (lō' 'êlōah), ainsi que par les qualificatifs à fonction dérogatoire qui suivent : «inconnus » (lō'yadā ûm) et «nouveaux » (hădāšîm). Enfin, le rapprochement entre les šêdîm bibliques et les šdyn de Deir 'Alla passe par des reconstructions linguistiques forcées qui ne sont, par ailleurs, attestées à aucun stade de la tradition manuscrite. La dérivation šedu> šd / šyd demeure donc la moins problématique, que ce soit d'un point de vue morphologique ou sémantique, et, dans ce cas, elle semble également être la plus vraisemblable ${ }^{55}$.

\section{La LXX et les cultes hellénistiques}

Le rôle des šêdîm dans le Chant de Moïse ayant été clarifié, nous pouvons maintenant regarder plus en détail la Lxx, à l'aide d'un double questionnement. D'une part, nous chercherons à comprendre quelles représentations sont mobilisées par le texte grec et par le vocabulaire qui y est utilisé, notamment en rapport à la sémantique du $\delta \alpha i \mu \omega v / \delta \alpha u$ óviov grec discuté dans le deuxième chapitre. D'autre part, nous nous poserons la question des pratiques rituelles qui pourraient constituer l'arrière-plan de ces passages. À cette fin, nous commencerons en reprenant la traduction grecque de Deut 32,17 :

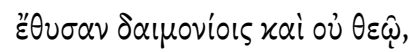

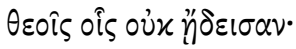

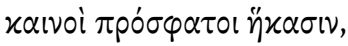

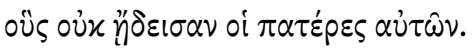

Ils firent des sacrifices à des démons et non à dieu,

à des dieux, qu'ils n'avaient pas connus, (des dieux) nouveaux, récents sont arrivés, que leurs pères ne connaissaient pas.

Brian Schmidt a discuté la question du rapport entre les šédîm bibliques et les šedu mésopotamiens, en soutenant, comme je le fais ici, la dérivation linguistique des šêdîm et des šedu et en refusant le parallèle avec les šdyn de Deir 'Alla (B. Schmidt 2016, p. 172186 et 2017). En revanche, je ne partage pas sa compréhension des šêdîm comme des « dieux-Shedu», à savoir des gardiens protecteurs ou des êtres intermédiaires bienveillants d'origine proche-orientale qui auraient été déifiés par une partie de la société israélite pour recevoir des sacrifices. En Mésopotamie, les šedu ne sont pas exclusivement des esprits protecteurs, mais ils peuvent souvent avoir un caractère malveillant prononcé (et, en ce sens, ils ne sont pas des intermédiaires, voir supra, p. 37). En outre, rien ne confirme la réalité d'un culte rendu aux šédîm / « dieux-Shedu » dans l'Israël ancien. Comme dans le cas du culte aux «boucs » que j'ai discuté dans le chapitre précédent, l'attitude polémique du discours sur le culte rendu aux démons mené par Deut 32 et par d'autres textes ne doit pas nécessairement être motivée par une réalité cultuelle. Elle relève plutôt de raisons d'ordre idéologique. 
Nous avons déjà observé en quoi la notion de $\delta \alpha i \mu \omega \nu$ en grec était beaucoup plus vaste et articulée que celle de «démon». Néanmoins, nous avons également remarqué que le terme se spécialise surtout à la période hellénistique pour indiquer une classe de puissances divines intermédiaires, distinctes des dieux et proches de la catégorie des héros. Une telle notion est développée davantage par la philosophie platonicienne et post-platonicienne, mais trouve des parallèles dans d'autres traditions philosophiques et religieuses grecques, notamment pythagoriciennes. Ces traditions sont particulièrement difficiles à dater car l'histoire de leur tradition manuscrite est très complexe. Bien qu'elles aient généralement été considérées comme très anciennes, la majorité des témoignages dont nous disposons ne date pas d'avant l'époque hellénistique. À cet égard, il vaut la peine d'ajouter au dossier que nous avons vu dans le deuxième chapitre un passage des «Commentaires » ou «Mémoires » pythagoriques, connus par Alexandre Polyhistor via Diogène Laërce ${ }^{56}$ :

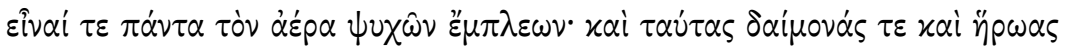

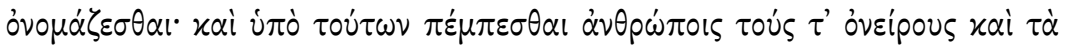

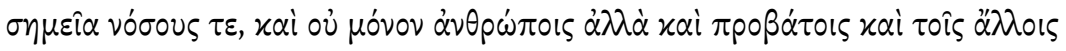

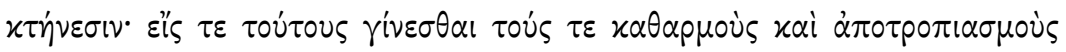

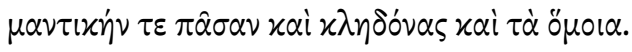

Tout l'air est rempli d'âmes. On les appelle démons et héros, et ce sont eux qui envoient aux hommes les songes et les signes et les maladies, et pas seulement aux hommes, mais aussi aux moutons et au reste du petit bétail. C'est à ces démons que l'on adresse les purifications et les rites de détournement, et toute la mantique et les sorts et les choses du même genre.

Diogène explique avoir tiré ces informations du traité sur la succession des philosophes écrit par Polyhistor, historien grec de l'époque de Sylla. Ce dernier déclare, à son tour, les avoir lues dans les Mémoires pythagoriques. Il est pourtant difficile d'établir le véritable noyau ancien de ces informations de troisième main. Cependant, de telles croyances ne sont pas nées de manière soudaine : comme nous l'avons vu, certaines remontent probablement jusqu'à

56 Commentaires pythagoriques ap. Alexandre Polyhisthor, in D.L. 8, 32 [ed. Delatte, trad. Détienne 1963]. Pour une distinction hiérarchique entre dieux et daimones, héros et ancêtres attribuée à Pythagore, voir ibid. 8, 23. Pour un commentaire à ce passage, voir Detienne 1963 , p. $3^{2-37}$, qui est, toutefois, très optimiste sur la possibilité que ces textes contiennent des théories pythagoriciennes remontant effectivement au $\mathrm{VI}^{\mathrm{e}}$ siècle avant notre ère. Pour une approche plus prudente, voir Sfameni Gasparro 1997. 
Hésiode. En outre, le rôle qui est ici attribué aux démons dans la pratique oraculaire est déjà attesté dès la fin de l'époque classique, du moins par les lamelles de Dodone ${ }^{57}$. La concentration de témoignages à partir de la période hellénistique demeure, néanmoins, suffisamment significative pour en conclure que c'est à partir de cette époque que les $\delta \alpha$ í $\mu$ oveৎ occupent un rôle de premier rang dans les débats religieux. Dans le passage des Mémoires pythagoriques deux éléments revêtent un intérêt particulier pour mon propos : tout d'abord, l'idée

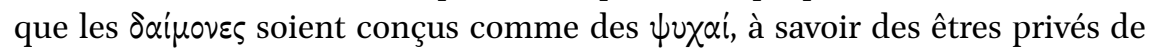
corps qui « remplissent l'air ». La relation entre $\delta \alpha i ́ \mu o v \varepsilon \varsigma$ et $\psi v \chi \alpha i$ est, d'ailleurs, exploitée également chez les philosophes juifs, comme le montre un fameux passage du livre sur les géants de Philon d'Alexandrie où il explique la nature

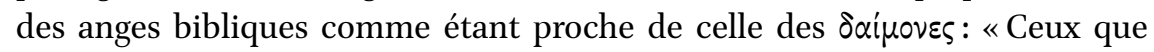
d'autres philosophes appellent 'démons' ( $\delta \alpha$ ípovas), Moïse les appelle d'habitude 'anges' : il s'agit d'âmes ( $\psi v \chi \alpha i$ i) qui volent à travers l'air ${ }^{58}$. Un deuxième élément suscitant l'intérêt est le fait que les démons soient destinataires de rites qui se différencient de la pratique sacrificielle régulière, comme les rituels

\section{$57 \quad$ Voir supra, p. 61.}

$5^{8}$ Philo, Gig. 6. Comparer, en outre, avec Somn. 1, 134-141. Comme ceci a été remarqué par Valentin Nikiprowetzky (1980) et Francesca Calabi (2004), Philon donne une relecture platonicienne du récit de la naissance des géants en Genèse 6 , sans accueillir les explications qui mettaient en relation la naissance des géants avec les anges déchus et représentaient une exégèse en vogue à l'époque, voire même le mythe fondateur de la démonologie du Second Temple (voir infra, § 8.1.1.). Il tient, au contraire, à souligner que les figures de la médiation dans les traditions grecques et juives sont largement

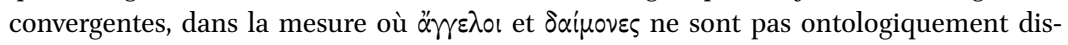

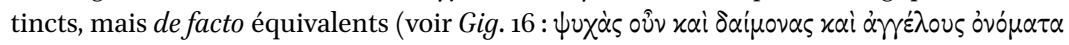

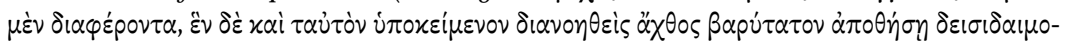
víav. «Donc en réalisant que démons et anges sont des noms différents mais qu'ils renvoient à une seule et même réalité, tu déposeras le fardeau très lourd de la superstition », et comparer avec Calabi 2004, p. 93-94). Une telle position est considérablement proche de la position de Plutarque dans le traité De la superstition. Il est, en tout cas, possible que Philon eût connaissance des traditions hénochiques sur les anges veilleurs, mais qu'il les ait intentionnellement rejetées dans son refus plus général de la démonologie comme croyance du «vulgus » (oi $\pi \circ \lambda$ i $i)$. On peut comprendre dans ce contexte la suite du pas-

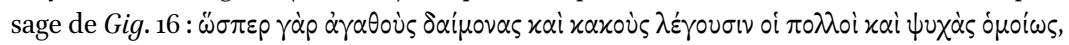

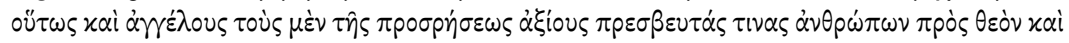

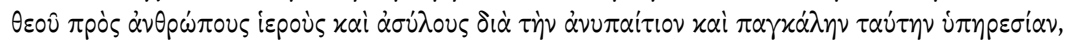

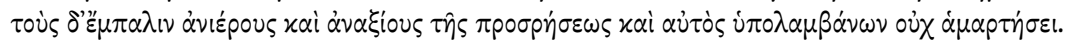
«Car, tout comme la plupart des gens croit dans des bons et des mauvais démons, et ainsi pour les âmes, de la même manière toi-même tu ne te tromperas pas lorsque tu estimes que, parmi les anges, certains, dignes de leur nom, sont ambassadeurs des hommes chez Dieu et de Dieu chez les hommes, sacrés et inviolables en raison de ce service sans défaut et magnifique, alors que d'autres, au contraire, sont impies et indignes de leur appellation »). Voir, à ce sujet, A.T. Wright 2005 a. 
apotropaïques, la divination et les purifications rituelles ${ }^{59}$. Ces deux aspects offrent deux points de comparaison pertinents avec Deut 32 :j'y reviendrai.

Il faut cependant préciser que, dans la LXX, le terme utilisé n'est pas $\delta \alpha i ́ \mu o v \varepsilon \varsigma$,

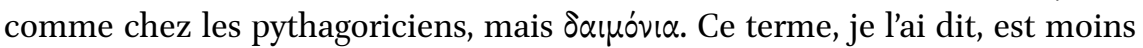
riche du point de vue des champs sémantiques recouverts (il couvre rarement, par exemple, l'idée de destin), néanmoins, il conserve une forte ambiguïté ainsi qu'une idée d'indétermination. L'Apologie de Socrate, dans laquelle le pilier de l'accusation est une prétendue différenciation entre les « dieux traditionnels » et les $\delta \alpha \iota o^{v} \iota \alpha$ introduits par Socrate, demeure le meilleur exemple de la double connotation que pouvait revêtir ce $\operatorname{mot}^{60}$. Toutefois, les similitudes entre les expressions employées par Platon et celles de Deut 32,17 (notamment

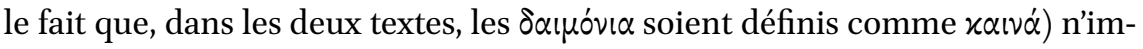
pliquent pas nécessairement qu'une influence platonicienne soit effective sur le traducteur61. Elles suggèrent, par contre, que le fait de qualifier un culte ou une divinité comme «nouvelle » ait été une stratégie de délégitimation répandue dans l'Antiquité. En outre, elles attestent le fait que le mot $\delta \alpha \mu o ́ v i \alpha ~ p o u v a i t$ être utilisé à l'occasion en opposition non seulement à $\theta \varepsilon o i ́$, mais également à $\delta \alpha i \mu o v \varepsilon \varsigma$, avec une intention déprécatoire. Ce procédé est bien exploité par le traducteur du Deutéronome. Dans le texte grec de Deut 32,16-17, il n'est plus question d'insister sur la nature «non divine » ou « moins divine » des démons : l'expression hébraïque $l_{\bar{o}}^{\prime}$ 'ělōah a été comprise comme indiquant un nom de Yhwh et a été traduite par un datif ( $0 \dot{v} \theta \varepsilon \hat{\omega})$, qui ne correspond probablement

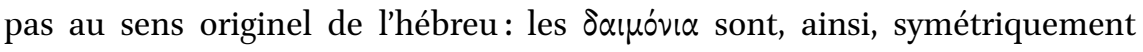

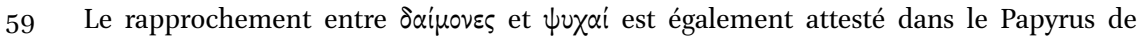

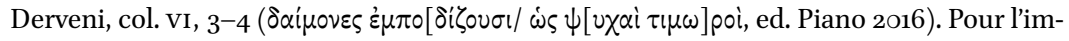
matérialité des $\psi v \chi \alpha$ í, voir Hp., Vict. 29 (Littré 6, p. 504-505); Pl., Phd. 114b-c.

6o Voir supra, § 2.3.

61 La possibilité d'une influence platonicienne sur le travail des traducteurs, notamment dans le Pentateuque, est un sujet débattu. Alors que Martin Rösel (1994) et Evangelia Dafni (2006) estiment que les traducteurs du Pentateuque font intentionnellement recours à des notions platoniciennes, d'autres chercheurs suggèrent la prudence (Van der Louw 2007, p. 83-84; Van der Meer 2016). Nous avons quelques témoignages papyrologiques sur la présence de Platon aux niveaux les plus hauts de l'éducation aux époques hellénistiques et romaines (Dupertuis 2007, p. 8-11). Certes, les auteurs de livres plus tardifs comme la Sagesse ou les Maccabées entrent en dialogue avec plusieurs idées de la tradition platonicienne et post-platonicienne. Cependant, une référence directe à l'Apologie de Socrate par les traducteurs qui étaient actifs au troisième siècle avant notre ère est, à mon sens, douteuse et loin d'être démontrée. Malgré la popularité du personnage de Socrate, l'Apologie n'était pas un texte d'école. Sur le canon de base de l'éducation dans l'Égypte gréco-romaine (qui comprenait Homère, Hésiode, Euripide, Ménandre et quelques orateurs), voir Morgan 1998, p. 67-73; Cribiore 2001, p. 178-205. 
opposés à Yhwh ${ }^{62}$. En outre, lorsque nous considérons l'ensemble du Chant de Moïse dans la LXx ancienne, nous repérons ci la toute première trace d'une opposition nette entre anges et démons, à savoir entre les vioi $\theta \varepsilon \circ \hat{v} / \alpha \gamma_{\gamma} \varepsilon \lambda \circ$

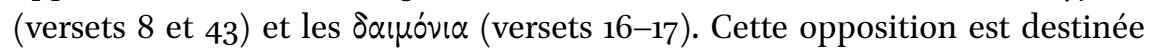
à acquérir un poids considérable dans les traditions juives et chrétiennes du début de notre ère.

\subsection{Un certain goût du sang}

L'idée que les démons reçoivent des cultes illégitimes trouve un développement ultérieur dans d'autres textes bibliques, qui viennent préciser en quoi consistent les sacrifices qui leurs sont dédiés. Un passage important en ce sens est Ps 105,37 LXX (= 106,37 TM) dans lequel il est question des mauvaises pratiques que les Israélites ont appris de leurs voisins cananéens, parmi lesquelles figurent la vénération d'images sculptées et les sacrifices d'enfants. À l'intérieur de ce discours les démons sont mentionnés comme destinataires de sacrifices humains qui deviennent une source de souillure à cause du sang versé. Puisque le grec traduit très littéralement l'hébreu, je me concentre sur le texte de la LXX sans discuter le $\mathrm{TM}^{63}$ :

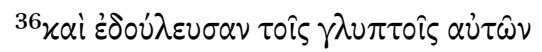

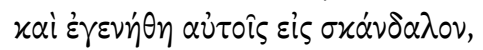

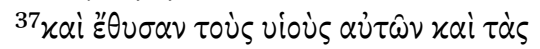

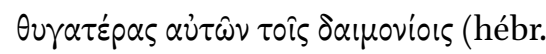
šedîm),

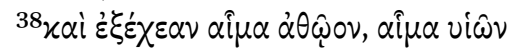

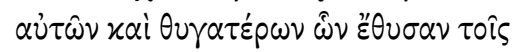

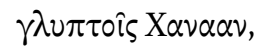

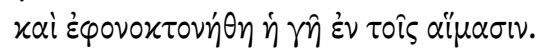

${ }^{36}$ Et ils s'assujettirent à leurs images et cela fut pour eux un piège, 37ils sacrifièrent leurs fils et leurs filles aux démons, ${ }^{38}$ et ils versèrent du sang innocent, sang de leurs fils et de leurs filles qu'ils avaient sacrifiés aux images sculptées de Canaan, et la terre fut souillée par le meurtre dans le sang.

La critique des images sculptées ainsi que la condamnation du sacrifice humain comme pratiques typiquement cananéennes sont des thèmes bien

62 '̌́lōah est une formation tardive qui peut renvoyer à Yhwh (par exemple en Job 3,4; Neh 9,17), ou indiquer génériquement une divinité (par exemple en Ps 18,31; Dan 11,37$38)$ : voir à ce sujet, entre autres, Heiser 2008, p. 138. L'ambiguïté était peut-être intentionnelle en hébreu. La technique du traducteur reflète un souci d'harmonisation qui s'étend à l'intégralité du verset et dans lequel le difficile śsāûum hébreu (dérivé la racine

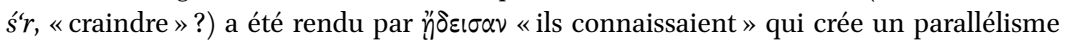
avec la moitié précédente du verset (voir Dogniez 1992, p. 330).

63 Ps $105,36-38(=106,36-38$ TM $)$. 
attestés dans la Bible et il n'est pas surprenant qu'ils soient associés ici. La question est plutôt de comprendre le rôle que vient jouer le thème du sacrifice aux démons dans ce contexte.

Du point de vue grec, ce passage rappelle les $\varphi \alpha \hat{\nu} \lambda \alpha \delta \alpha \_\mu o ́ v i \alpha$, les démons malveillants décrits par Plutarque dans le traité Sur la disparition des oracles et dans d'autres textes ${ }^{64}$. D'après Plutarque, l'idée selon laquelle les démons apprécient les sacrifices humains, et tout particulièrement ceux de jeunes gens, est répandue, même si elle ne devrait recevoir aucun crédit. Un passage de la vie de Pélopidas est assez explicite en ce sens. Plutarque raconte qu'un dieu apparut en rêve à Agésilas, le chef de l'armée de Sparte, et lui demanda, à l'image d'Agamemnon, de sacrifier sa fille pour assurer le succès de son expédition, ce que le chef refusa de faire. À propos de cet épisode, Plutarque nous dit que plusieurs considèrent qu'un sacrifice aussi impie ne peut, en aucune manière, relever de la volonté des dieux, ici définis comme «les êtres supérieurs », mais doit nécessairement appeler en cause des démons ${ }^{65}$ :

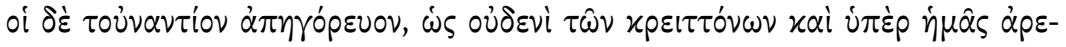

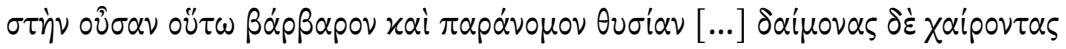

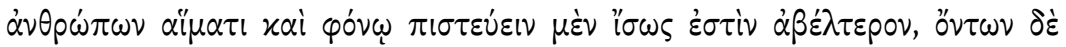

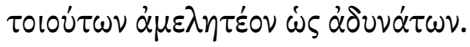

Les autres étaient d'un avis contraire et soutenaient qu'un sacrifice si barbare et si criminel ne pouvait être agréable à aucun des êtres supérieurs de qui nous dépendons [...] il est stupide de croire à l'existence des démons qui prennent plaisir au meurtre et au sang des hommes, ou, s'ils existent, il faut les négliger comme étant sans puissance.

Dans ce passage reviennent deux idées également présentes dans la LXX : celle selon laquelle des sacrifices humains sont offerts à des démons et celle qui prétend que les démons, dont l'existence est disputée, sont des puissances

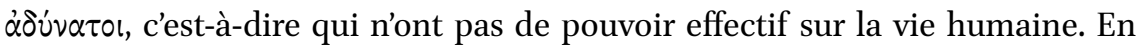
revanche, l'insistance sur l'élément de souillure impliqué par ces sacrifices est une caractéristique typiquement biblique qui n'a pas de parallèle dans les

64 Voir Plut., Def. orac. 417 b et supra, § 2.3-2.4.

65 Plut., Pel. 21, 5-6 (trad. Flacelière et Chambry, Paris, Les Belles Lettres, 1966). Comparer, également, avec Def. orac. (417c-d) et voir, pour ce passage, Georgiadou 1997, p. 166-171. Pour un commentaire sur la position de Plutarque vis-à-vis de ces croyances, voir Brenk, 1977, p. 55, 1998, p. 177. 
récits plutarchéens. Elle apparaît encore dans un passage de la LXX d'Isaïe, chapitre 65 , versets $3^{-4}$, qui nous décrit des rituels adressés aux démons ${ }^{66}$ :

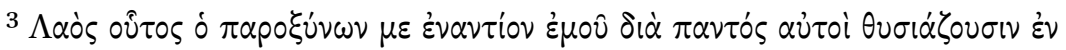

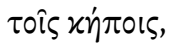

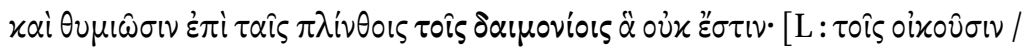

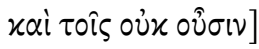

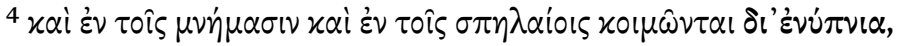

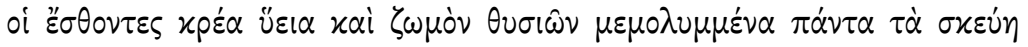
$\alpha \dot{\tau} \tau \hat{\omega}$.

${ }^{3}$ Ce peuple, qui m'exaspère en ma présence tout le temps, ce sont eux qui sacrifient dans les jardins,

et qui brûlent de l'encens sur les briques pour les démons - qui n'existent pas.

4 Dans les sépulcres et dans les grottes ils dorment pour des songes,

eux qui mangent la viande de porc et la sauce des sacrifices - tous leurs objets sont souillés.

Il faut observer que tant la mention des démons que celle des rêves sont absentes du texte hébreu qui lit:

${ }^{3}$ Ce peuple qui me provoque $(k s)$, devant ma face, continuellement, ceux qui font des sacrifices dans les jardins et qui font monter la fumée sur les briques (ûmaqatțarîm 'al hallabēnîm),

${ }^{4}$ ceux qui se tiennent (hayyōšabîm) dans les tombes et passent la nuit dans des lieux cachés,

Mangent de la viande de porc et du brouet souillé dans leurs pots.

Les expressions hébraïques et grecques qui décrivent ici l'offense à Yhwh ressemblent à celles employées en Deut 32 (par exemple, l'emploi du verbe $k$ 's/ $\pi \alpha p \circ \xi \dot{v} v \omega$, ici et en Deut 32,16 ) et sont typiques du langage deutéronomique ${ }^{67}$.

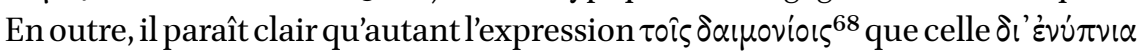
sont des ajouts imputables au traducteur et qu'ils ont une fonction exégétique, à savoir qu'ils visent à clarifier les destinataires et le but des rituels décrits qui sont assez obscurs en hébreu. Il se peut que la référence aux démons ait

66 J'adopte ici la traduction d'Alain le Boulluec et Philippe Le Moigne, Paris, Cerf, 2014.

67 Voir, par exemple, Deut 4,25; 9,18 ; 31,29; comparer également avec Ps 78,58 (= 77,58 LXX).

68 Pour la critique textuelle de ce passage, voir supra, p. 82. 
effectivement été inspirée au traducteur par le passage de Deut 32,16. Il est, en revanche, difficile d'identifier les pratiques auxquelles il est fait allusion dans le texte hébreu d'Is 65,3-4. D'après la proposition de Susan Ackerman, les « sacrifices dans les jardins » pourraient indiquer un culte pour la déesse Asherah, vu que l'association entre jardins et arbres sacrés apparait déjà en Is 1,29 et que l'arbre est une représentation traditionnelle de cette déesse ${ }^{69}$. Une autre hypothèse est d'y voir une référence au dieu de la végétation Adonis-Tammuz dont le culte était populaire au début de l'époque hellénistique, ce qui correspond à une datation parfois assignée au troisième Isaïe ${ }^{70}$. Pour ce qui est des rituels accomplis sur les briques, la référence est encore plus obscure. Bernhard Duhm suggérait déjà que l'hébreu labēnâ pouvait indiquer un autel de terre ${ }^{71}$. Le rituel est compris d'habitude comme une offrande d'encens, d'après la signification attribuée à la forme piel du verbe $q t ̦$, littéralement « faire monter la fumée $»^{72}$. L'interprétation est probable mais non assurée car, ailleurs, la même racine peut faire référence à la fumée produite par le sacrifice ${ }^{73}$. Le grec utilise ici l'équivalent usuel de $q t$ tr Piel, $\theta u \mu i \alpha \omega \omega$, «brûler de manière à produire de la fumée » qui correspond bien à la signification du verbe hébreu et dont l'usage pour les encens et les parfums est bien attesté en grec.

Il paraît cependant improbable que le traducteur ait reconnu Asherah ou Tammuz comme étant les destinataires des rites mentionnés au verset 3 . En tout cas, rien dans la formulation ne le laisse entendre. En revanche, la référence aux jardins, aux briques et à l'encens pourrait lui avoir suggéré l'idée d'un rituel de détournement pour les démons, probablement accompli à proximité des maisons. À cet égard, il faut aussi rappeler que la tradition lucianique est partagée en ce qui concerne la tradition manuscrite du verset $3 \mathrm{~b}$.

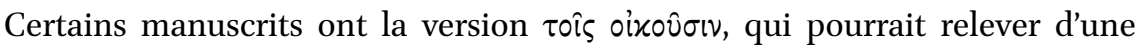
tentative de rendre l'hébreu hayyôšabîm au début du verset 4, lequel ne serait

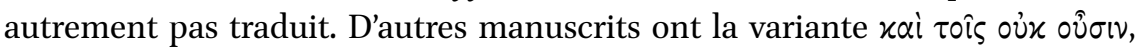
qui a une signification similaire à $\ddot{\alpha}$ oủx है $\sigma \tau \iota^{74}$. J'aurais tendance à considérer cette dernière lecture comme relevant de la Septante ancienne, alors que

69 Ackerman 1992, p. 165-212. Voir également Is 66,17. En Is 1,29 il est question de êlîm (térébinthes) et jardins : ici le traducteur comprend êlîm comme «idoles ».

70 Ainsi Eissfeldt 1970, p. 19-20. Tammuz est également mentionné en Ez 8,14 : voir Ribichini 1999 a, p. 9. Pour d'autres références au culte d'Adonis-Tammuz dans le livre d'Isaïe, voir Delcor 1978 (Is 17); Bonnet 1987 (Is 15).

71 B. Duhm 1892, p. 475 ; suivi par Westermann 1966, p. 318.

72 Ainsi Westermann 1966, p. 318; Watts 1985-1987, p. 339 ; Ackermann 1992, p. 169-174; Blenkinsopp 2003, p. 266-267.

73 Pour une discussion de cette racine, voir Angelini 2019, p. 249, note 3 o.

74 Voir Ziegler 1939, p. 36o, apparatus I. 


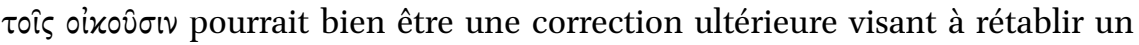
texte plus proche de l'hébreu et soulignant encore le caractère domestique de ces cultes ${ }^{75}$.

La référence à des rituels spécifiques pour les démons et la mention de la divination par les songes suggèrent que le traducteur d'Isaïe se situe dans un registre de croyances et de pratiques destinées aux démons similaire à celui des textes d'Alexandre Polyhistor et de Plutarque. La référence aux «démons qui n'existent pas » pourrait, en outre, suggérer que le traducteur s'inscrit dans un débat philosophique courant à son époque. Il faut également tenir compte du fait que, parmi les lettrés de l'époque hellénistique, il n'est pas rare d'inter-

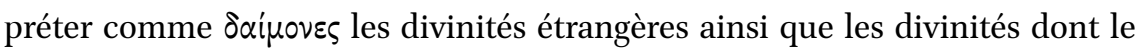
culte est attesté en Grèce mais qui sont considérées comme provenant de l'extérieur. Pour le premier cas, l'on peut penser à Osiris, Isis et Typhon cités chez Plutarque qui ne peuvent pas être considérés comme des dieux à part entière à cause de la nature «trop corruptible» de leurs vicissitudes, telles qu'elles sont connues par les mythes ${ }^{76}$. Dans le deuxième cas rentrent, entre autres, les dieux thraces et phrygiens nommés par Strabon, tels que Sabazios ou la Grande Mère, mais également les nymphes, les satyres et les Courètes. Les

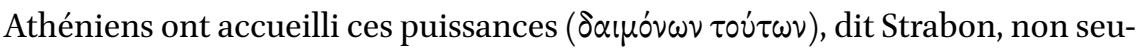
lement comme des servants des dieux ( $\pi$ pó $\pi \circ \lambda \circ \imath \theta \varepsilon \hat{\omega} v)$, mais également comme de véritables divinités $\left(\alpha\right.$ viroi $\theta \varepsilon$ coi $^{77}$. Là encore, je ne songe pas à une influence directe de textes philosophiques ou ethnographiques grecs sur les traducteurs de la Bible hébraïque. Je tiens plutôt à souligner l'importance de la LXX en tant que premier témoin d'une notion religieuse qui sera ensuite répandue ailleurs dans le monde hellénistique. Les similitudes de langage sont ainsi l'indice d'une sensibilité religieuse nouvelle et partagée qui peut également servir des buts différents, voire divergents entre eux.

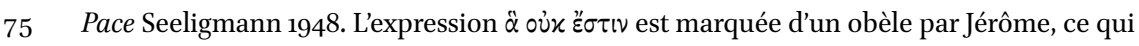
indique sa présence dans la Septante ancienne. Le caractère mouvant de ce verset dans l'Antiquité est encore confirmé par une lecture assez obscure conservée par un manuscrit qumrânien : וינקו ידים על האבנים, «et ils vident leurs mains sur les pierres » (1QIsaa, Ulrich et Flint 2010, DJD 32, vol. 2, p. 190). Le sens de l'expression n'est pas clair (voir encore Parry 2019, p. 430), mais Frank Moore Cross suggérait qu'elle pouvait faire référence à un rituel d'encens où yad (littéralement, «main ») aurait ici eu le sens de kaf, «le plat» de la main, mais également celui de la mesure pour une cuillère d'encens (Cross, cité par Hanson 2000, p. 141).

76 Plut., Is. Os. 355e-36of, notamment 36oa. Chez Plutarque, Typhon est le nom grec du dieu Seth. Les croyances des Égyptiens sont définies par Plutarque comme impies et barbares

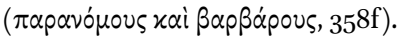

Str. 10, 3, 18-19 (C 471-472). 
Le discours mené dans les textes bibliques, à la fois en hébreu et en grec, est d'ailleurs caractérisé par une violence polémique qui n'a pas de véritables parallèles dans le monde grec. Cette manière explicite d'exprimer la polémique contre les dieux étrangers pour véhiculer un discours identitaire reste propre aux traditions bibliques: en ce sens, ce n'est pas une innovation des traducteurs, mais cela concerne déjà le texte de départ ${ }^{78}$.

De ce point de vue, lidée que les démons n'existent pas a, également, des résonnances à l'intérieur de la tradition biblique elle-même, notamment car, par ce biais, le traducteur associe aux démons un motif qui était typique de la polémique contre les idoles, à savoir le fait qu'ils sont dénués de pouvoir. Alors que la polémique contre les idoles insiste surtout sur leur matérialité inutile, dans le texte d'Isaïe la faiblesse des démons est suggérée par un manque de substance ${ }^{79}$. La vénération des idoles est encore associée aux cultes aux démons dans d'autres traditions du Second Temple, comme l'atteste un passage du livre d'Hénoch où les démons sont mis en parallèles avec les «fantômes » $(\varphi \alpha \nu \tau \dot{\alpha} \sigma \mu \alpha \tau \alpha)$ et présentés comme sources d'impuretée ${ }^{80}$ :

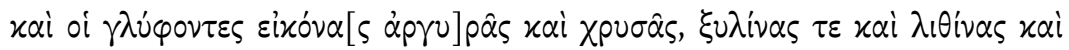

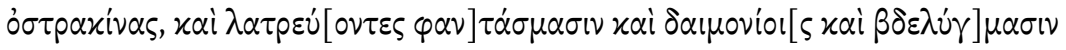

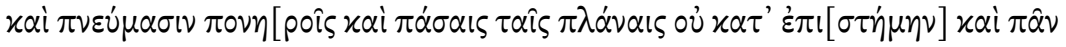

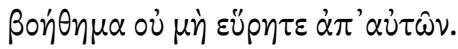

Et ceux qui gravent des images en argent, en or, en bois, en pierre et en argile et adorent des fantômes, des démons, des êtres abominables, des esprits mauvais et toutes sortes d'erreurs, faute de connaissance, et vous ne recevrez d'eux aucune aide.

Ce rapprochement entre démons et idoles est donc un thème qui mérite d'être analysé plus en détail. Avant de s'y pencher, il faut toutefois s'arrêter brièvement sur un dernier passage de la LXX d'Isaïe où il est encore question d'un culte «démoniaque », ou pour mieux dire « daïmonique», à savoir Is 65,11.

78 Sur le sujet et sur sa diffusion chez les auteurs chrétiens, voir Anderson 2016 ; Barbu 2016, p. $40-88$.

79 Voir également Van der Vorm-Croughs 2014, p. 468-469. Sur le discours sapientiel contre les idoles dans la Bible hébräque, voir récemment Ammann 2015. Des formulations

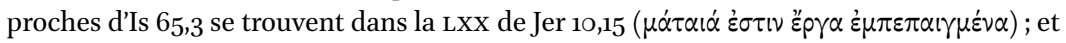
d'Hab 2,18 ( $\chi \dot{\omega} \omega \varepsilon \varepsilon u \mu \alpha, \varphi \alpha \nu \tau \alpha \sigma i \alpha \nu ~ \psi \varepsilon v \delta \hat{\eta})$.

8o 1 Hen 99,7 d'après la version du papyrus Chester Beatty. 


\subsection{Retour sur Bon démon et Fortune}

J'ai déjà annoncé dans le troisième chapitre que Is 65,11 constituait un cas de figure en partie différent par rapport à la thématique des dieux des autres ${ }^{81}$. Dans ce passage, la polémique est adressée à ceux qui « préparent la table pour

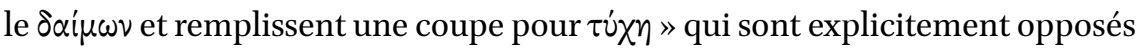
aux « servants de Yhwh» (verset 8) et sont donc destinés à une très mauvaise fin (verset 12a) $)^{82}$ :

\begin{tabular}{|c|c|}
\hline 11 & 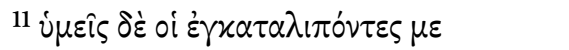 \\
\hline השכחים את הר קדשי & 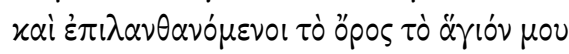 \\
\hline הערכים לגד שלחן & 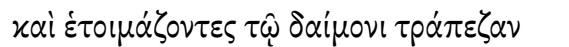 \\
\hline והממלאים למני ממסך & 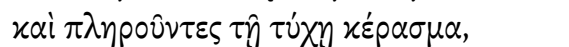 \\
\hline 12 ומניתי אתכם לחרב וכלכם לטבח תכרעו & 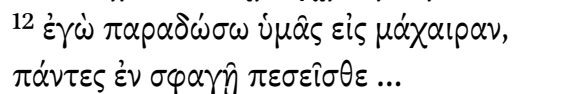 \\
\hline
\end{tabular}

${ }^{11}$ Mais vous qui abandonnez Yhwh,

${ }^{11}$ Mais vous, qui m'abandonnez, qui oubliez mon mont saint, qui oubliez mon mont saint, Qui préparez une table pour Gad, et remplissez un mélange (de vin) pour Méni

12 Je vous ai destinés à l'épée, qui préparez une table au démon et remplissez une coupe à la Fortune, 12 moi je vous livrerai à l'épée, vous tomberez tous immolés ... et vous tous vous mettrez à genoux pour l'abattage ...

Au vu de la variété de lectures attestées par la tradition manuscrite, j’ai décidé

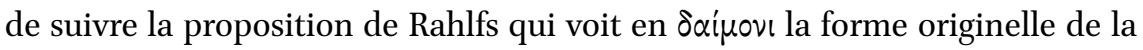
Septante ancienne. Nous trouvons ici la seule occurrence de $\delta \alpha i \mu \omega \nu$ au lieu

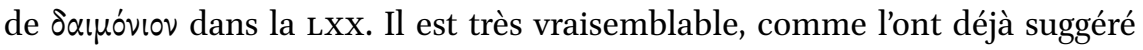
Seeligmann et ensuite Schaper ${ }^{83}$, que le traducteur ait interprété les sacrifices offerts à Gad et Méni comme des références aux cultes de la «Bonne Fortune » et $\mathrm{du}$ «Bon démon» (agathetyche et agathodaimon): deux puissances bienfaisantes et protectrices extrêmement populaires dans tout le bassin méditerranéen aux époques hellénistique et romaine et souvent nommées ensemble. Même si l'on reste toujours dans le cadre de la polémique contre les dieux étrangers, Schaper a donc raison de souligner que la mention du $\delta \alpha i ́ \mu \omega \nu, i c i$,

\footnotetext{
81 Pour une discussion des variantes textuelles, voir supra, p. 82-83.

82 Is 65,11-12a. Pour le grec, je reprends la traduction d'Alain Boulluec et Philippe Le Moigne, Paris, Cerf, 2014.

83 Seeligmann 1948, p. 264-265 [99-100]; Schaper 2010.
} 
ne vise pas à « démoniser » les dieux des autres, mais qu'elle correspond à une référence cultuelle précise ${ }^{84}$.

Par contre, quelques aspects de l'interprétation de ce passage méritent d'être discutés plus en détail. Le premier concerne la dialectique entre dimension publique et privée du culte rendu au bon démon, qui semble être particulièrement complexe en Égypte ${ }^{85}$. Il est, en effet, indéniable que l'agathodaimon a joué un rôle important dans les récits de fondation d'Alexandrie et que, dans cette ville, son culte a eu une portée civique considérable. D'un autre côté, plusieurs sources identifient l'agathodaimon à différentes divinités indigènes et anciennes d'Égypte et son nom était également appliqué à un bras du fleuve $\mathrm{Nil}^{86}$. D'après Schaper, le traducteur aurait eu le double objectif de mettre en garde son public à la fois contre la participation aux rituels civiques pour $\delta \alpha i \mu \omega \nu$ et $\tau \dot{x} \chi \eta$ et contre le danger représenté par la pénétration du culte dans les maisons juives d'Alexandrie. Il me semble, toutefois, que la dimension publique de ce culte n'est jamais mise en avant par le texte. La référence à la coupe et à la table souligne plutôt l'aspect domestique du rite qui est, sans doute, le plus ancien et qui est également bien attesté dans les sources grecques dès l'époque classique où les libations au «bon démon » et au «bon sort» inauguraient traditionnellement les symposiums et sont bien attestées en contexte dionysiaque ${ }^{87}$. En outre, la nature chtonienne souvent attribuée à l'agathodaimon, vénéré en Égypte sous la forme d'un serpent ${ }^{88}$, s'inscrit bien dans le contexte plus large du chapitre 65, versets 1-11, puisque dans les versets $3^{-4}$ il est fait référence à des rituels qui avaient lieu dans des jardins et dans des grottes. De ce point de vue, la mention du démon au verset 11 fait écho, voire reprend probablement de manière intentionnelle celle du verset 3 où les

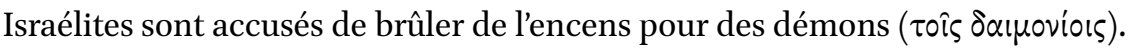

Cette remarque m'amène au point suivant, qui est d'ordre méthodologique. On a souvent utilisé Is 65,11 comme une démonstration de la nature profondément hellénisée et «actualisante » de la LXx d'Isaïe, nature qui ne peut en

$84 \quad$ Schaper 2010, p. 146, pace Riley 1999, p. 238.

85 Pour un répertoire des sources littéraires et épigraphiques, la référence est encore Ganschinietz 1918, p. 37-59; pour les sources iconographiques, Dunand (LIMC I-II, s.v.); sur le culte à Alexandrie, voir Visser 1938, p. 6-8; Fraser 1972, I, p. 209-211; Ogden 2014. Pour un traitement des sources hellénistiques, voir Sfameni Gasparro 1997. Les cultes du bon démon et Fortune sont mentionnés également par Philon (Prob. 39).

86 Voir Bernand 1969, no. 175, II, 9 ; Philon de Byblos chez Eusèbe (Praep. 1, 10, 48).

87 Les loci classici sont chez Aristophane (Eq. 85 et 105; Pax 300; v. 525). Pour d'autres sources voir Ganschinietz 1918, p. 40. Sur l'agathodaimon en contexte dionysiaque voir Pirenne-Delforge 2020 , d'après laquelle la référence à l'agathodaimon exprime la manifestation de la puissance de Dionysos dans le cadre du sympose.

88 Voir les sources citées par Dunand 1918, ainsi que Daszewski 1991, p. 96. 
aucune manière être remise en question. Cependant, la traduction d'Isaïe n'est pas seulement une traduction libre ou actualisante: comme le rappelle justement John Lee ${ }^{89}$, elle est davantage une traduction littéraire. Il faut donc envisager l'hypothèse que certaines innovations ne soient pas forcément à interpréter à la lumière d'un véritable conflit religieux (ou encore moins politique), mais qu'elles soient plutôt à expliquer par le choix d'un registre linguistique qui est hellénisant car élevé. Les deux références aux démons introduites aux versets 3 et 11, toutes deux absentes en hébreu, pourraient en partie être expliquées par des raisons d'ordre littéraire, à savoir un renvoi d'ordre stylistique voulu par le traducteur à l'intérieur du chapitre 65. En outre, ce dernier pourrait avoir choisi $\delta \alpha i \mu \omega \nu$ et $\tau \dot{x} \not \eta$ car ils étaient de facto les meilleurs équivalents pour Gad et Méni dans ce contexte, comme il me semble pouvoir le démontrer.

Un aspect moins discuté de ce passage concerne, effectivement, la relation entre le grec et l'hébreu. À cet égard, la question de la convergence entre les noms divins choisis par le traducteur et ceux mentionnés dans le texte hébreu n'a pas été suffisamment prise en compte. Méni est une puissance peu connue et ce passage représente sa seule occurrence dans la Bible hébraïque. Toutefois, la racine $m n h$, « diviser», « compter», et parfois «assigner» est bien attestée en hébreu et en ougaritique (mny, «assigner en sort») et des antécédents ont été repérés dans l'onomastique d'Ebla et d'Ougarit ${ }^{90}$. Ce verbe est considéré comme étant un emprunt à l'accadien manu, «repartir», «attribuer», « donner la part». Il est donc fort probable que Méni soit une divinité du sort, vraisemblablement à mettre encore en relation avec la déesse Manat attestée au Levant dès l'époque nabatéenne, notamment à Palmyre, et il est possible qu'elle ait été identifiée à $\tau \dot{x} \chi \eta / F o r t u n a$ dans les sources d'époque romaine ${ }^{91}$. D'ailleurs la reprise du verbe $m n h$ au verset 12 (ûmānittî, «je vous ai assignés », «destinés », mais à l'épée! dit Yhwh) semble jouer précisement sur cet aspect du dieu. Gad est en revanche une divinité bien connue dans le Levant. Elle est attestée dans la Bible hébraïque comme nom propre, comme théonyme

\section{$89 \quad$ Lee 2014 .}

$90 \quad$ Baldacci 1978. Le texte administratif d'Ebla est un décompte de portions d'orge qui contient le nom d'un fonctionnaire appelé «Mani a donné » (TM.75.G.336, verso I, 4, publié par Pettinato et Matthiae 1976, p. 5).

$91 \quad$ Elle est attestée épigraphiquement à partir du IV ${ }^{\mathrm{e}} \mathrm{s}$. av. J.-C. par une inscription cultuelle en Arabie du Nord et par deux théophores, et apparaît en outre dans une inscription funéraire nabatéenne (CIS II, 198) : voir les sources collectées par Roche 2014, p. 3-4. La déesse est, en outre, représentée comme Némésis avec le sceptre à Palmyre, et un buste de Tyché a été repéré au centre d'un zodiaque dans le temple nabatéen de Khirbet el Tannur. Sur le culte de Manat on peut voir également Fahd 2012, p. 373-374; Sperling 1999, p. 566-568; Gorea 2010, p. 156-157. 
et comme toponyme ${ }^{92}$, parfois avec une référence directe au bon sort ${ }^{93}$. Le nom est d'ailleurs présent dans l'onomastique du premier millénaire avant l'ère dans tout le Levant ${ }^{94}$. La documentation épigraphique date en grande partie de l'époque romaine et comprend une cinquantaine d'inscriptions, dont la plupart ont été retrouvées à Palmyre et Hatra ${ }^{95}$. Gad est également attesté en contexte punique par trois inscriptions provenant d'Ibiza, de Nora (Sardaigne) et de Mactar (Tunisie) ${ }^{96}$. Comme Ted Kaizer et, plus récemment, Ryan Thomas l'ont relevé, Gad endosse dans l'épigraphie plusieurs fonctions différentes; il peut être seul ou accompagné d'autres divinités (comme par exemple Ba'al et Tanit ${ }^{97}$ ); son nom peut être isolé ou suivi par un locatif ( $« \operatorname{Gad} \mathrm{de} \ldots{ }^{98}$ ) et il est souvent utilisé comme épithète plutôt que comme théonyme. Il pose, donc, le même ordre de problèmes que ceux soulevés par $\alpha \gamma \alpha \theta 0 \delta \alpha i \mu \omega \nu$ et par $\tau u ́ x \eta$, à savoir celui de l'articulation entre un qualificatif susceptible d'être assigné à plusieurs divinités et la désignation d'une entité divine indépendante. À ce propos, Ryan Thomas souligne justement que le concept de «personnification » ou « d'abstraction » est de loin trop simpliste pour expliquer l'articulation entre les deux niveaux ${ }^{99}$. La documentation épigraphique démontre, d'ailleurs, que ces deux usages ne doivent pas être considérés comme alternatifs, ni mutuellement exclusifs. Dans l'Antiquité, une telle ambiguïté peut caractériser n'importe quelle divinité, même si le phénomène semble être particulièrement prononcé pour les puissances divines liées au sort, autant au Levant qu'en Grèce. En outre, tout comme agathodaimon et Tyché, Gad a une double nature divine : d'un côté civique, patronne de la cité, de l'autre familiale ou personnelle, qui le rapproche d'un « bon génie ». Il n'est donc pas complètement identique à Tyché, bien que les deux notions, néanmoins proches, aient été, à un moment donné, confondues et superposées ${ }^{100}$. L'équivalence entre Gad et Tyché est explicitement attestée pour la première fois dans une inscription bilingue de Palmyre du premier siècle de notre ère, mentionnant Gad

92 Voir notamment Ba'al-Gad en Jos 11,17.

93 Notamment en Gen 3o,11 à propos du fils de Leah : « et Leah dit : 'quelle chance'! et appela son fils 'Gad' ».

94 Voir Thomas 2019.

95 Le dossier a été publié et analysé par Kaizer 1997, 1998.

96 Respectivement RES $1222 ;$ KAI 72 et 147. Une inscription provenant de Lachish et datant de l'âge du Bronze est douteuse : voir Ribichini 1999 b, p. 339-341.

$97 \quad$ KAI 72 b, traduite et commentée par Kaizer 1998.

98 Par exemple PAT 0179, O273, 1079, 1097, traduites et commentés par Kaizer 1997.

99 Thomas (2019, p. 311), d'après lequel Gad ne serait à l'origine qu'une épithète du dieu El.

100 Voir Kaizer 1998, p. 51-53; 58-59, et Roche 2014, p. 3. 
dans le texte araméen et $\tau u ́ x \eta$ en grec ${ }^{101}$. Dans ce cadre, la LXX d'Is 65,11 mérite d'être ajoutée au dossier en tant que premier témoignage de la perception des anciens d'une proximité parmi ces deux notions du divin. En outre, ce passage constitue la seule attestation dans la Lxx d'une acception du $\delta \alpha i \mu \omega \nu$ comme puissance liée au destin, correspondant probablement à la signification première et plus profonde du mot dans la culture grecque.

\section{3}

\section{Démons, images, idoles}

Revenons à présent sur la relation entre démons et idoles que nous avons vus associés dans le Ps 106 ainsi que dans des traditions du Second Temple, telles que le livre d'Hénoch. Le discours sur les prétendues idoles est en réalité déjà lié à la polémique contre les dieux étrangers dans le Pentateuque, notamment en Deut 32, au verset 21 :

Ils m'ont rendu jaloux par un non-dieu $\left(l \bar{o}^{\prime} ' \bar{e} l\right)$, Ils m'ont provoqué par leurs pratiques vaines (bəhablêhem), Et moi je les rendrai jaloux par un non-peuple.

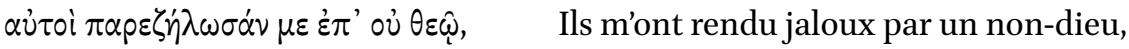

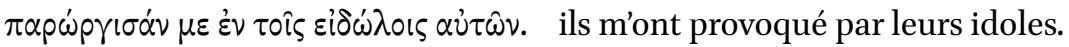

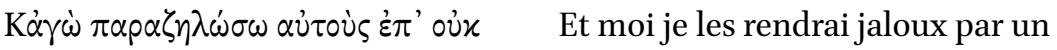
है$\theta v \varepsilon 1$, non-peuple,

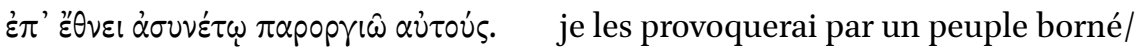
obtus.

Ce verset constitue le sommet de la polémique entamée au verset 16 et dans le texte grec les $\varepsilon^{\prime \prime} \delta \omega \lambda \alpha$ semblent occuper la même fonction que les $\delta \alpha \mu o ́ v i \alpha$ aux versets 16-17. Le grec عið $\omega \lambda \lambda \alpha$ traduit ici l'hébreu hăbālîm. L'équivalence entre hebel et $\varepsilon$ हiठ $\omega \lambda$ ov n'est pas un choix littéral, l'équivalent le plus littéral et le plus fréquent pour hebel dans la LXx étant $\mu \alpha \dot{\tau} \tau \alpha$ เos. Il s'agit, en revanche, d'un choix orienté vers la langue cible car le traducteur recourt à une notion grecque polysémique et extrêmement opérationnelle. Un aspect spécifique du champ sémantique de l'zî̉ $\omega \lambda \lambda_{0}$ en grec a probablement orienté ce choix, à savoir sa

101 CIS II, 3927. Transcription, traduction et commentaire en Kaizer 1997, p. 152-153. L'anthroponyme Eytychés est également attesté, transcrit en sémitique ('tks), sur un autel nabatéen (Milik 1976 b, p. 148). Sur les cultes de Tyché dans la Palestine d'époque romaine voir Belayche 2003 . 
nature trompeuse. Depuis Homère, le mot peut être appliqué à toute image qui restitue l'apparence d'un individu ou d'un objet qui est ailleurs ou qui n'est pas réel : l'image d'une personne qui apparaît en rêve (övap) $)^{102}$, de fantômes ou spectres du monde des morts $\left(\psi v \times \chi^{\prime}\right)^{103}$, ou une image créée exprès par les dieux $(\varphi \dot{\alpha} \sigma \mu \alpha)^{104}$; mais également l'ombre d'un corps $\left(\sigma \varkappa \iota^{\prime}\right)^{105}$, l'image reflétée par un miroir ${ }^{106}$, etc. Cet emploi du mot souligne le fait que la représentation visuelle produite par l'zi' $\delta \omega \lambda$ ov ne correspond pas à une réalité. Dans certaines sources, le manque de réalité correspond à un manque de corps: Lucien, par

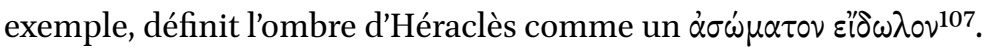

Si l'on compare ces usages à la signification originaire de hebel, «vapeur», d'où dérive l'idée de « vanité », « inutilité », sa traduction par عið $\delta \omega \lambda \alpha$ en Deut 32 semble refléter la conscience de la part du traducteur que le mot peut, parfois, renvoyer à quelque chose qui manque de substance ${ }^{108}$. Par ailleurs, la racine $h b l$ et les images provenant des rêves sont déjà associés dans quelques textes bibliques, comme par exemple Zach 10,2 qui condamne les songes comme moyen divinatoire car ils offrent seulement une consolation inutile (hebel). Un passage difficile du livre du Qohéleth compare les rêves (hălōmôt) aux vanités $(h a ̆ b a \bar{l} l i m)^{109}$. Cet arrière-plan pourrait également avoir justifié l'équivalence entre $\varepsilon \varepsilon^{\prime} \delta \omega \lambda$ ov et hebel en Deut 32,21110. Dans la péricope constituée par les ver-

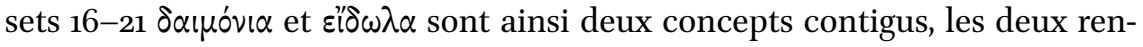
voyant à des entités privées de substance. On a vu qu'un tel rapprochement a ses parallèles dans les philosophies hellénistiques, et, dans sa biographie de Dion, Plutarque critique tous ceux qui croient aux apparitions de démons et de spectres $\left(\varphi \alpha \dot{v} \tau \alpha \sigma \mu \alpha \delta \alpha^{\prime} \mu \mu^{\prime} \nu \circ \varsigma \mu \eta \delta^{\prime} \varepsilon^{\prime \prime} \delta \omega \lambda \circ \nu\right)^{111}$. Dans la LXX ces deux concepts deviennent proches au point d'être interchangeables, comme cela est démontré par un passage du Ps $95(=96 \mathrm{TM})$ et par sa réécriture dans le chapitre 16 du premier livre des Chroniques ( 1 Paral 16 LXx). Le psaume célèbre la royauté de Yhwh et insiste sur la faiblesse des autres dieux lorsqu'ils sont comparés à Yhwh. L'hébreu lit ${ }^{112}$ :

\footnotetext{
102 Synesius cyr., De Somniis 15.

103 Hom., Od. 11, 471-476 ; 24, 14; Hdt. 5, 92.

104 Hom., Il. 5, 449-453; Od. 5, 796.

105 Aesch., A. 839.

106 Plut., Pyth. orac. 404c 10.

107 Luc., DMort. 11, 5.

108 Comme le suggère déjà Hayward 2007, p. 44-45.

109 Qoh 5,6.

110 Hayward 2007, p. 44-46.

111 Plut., Dion 2, 2-4, et supra, p. 76.

112 Ps 96,5 (= 95,5 LXX).
} 
כי כל אלהי העמים אלילים ויהוה שמים עשה

Tous les dieux des peuples sont des dieux de rien ('êlilîm), alors que Yhwh a fait les cieux.

1 Chroniques 16 est un chant attribué à David, composé par la réécriture de plusieurs psaumes combinés. Le verset 26 de ce chant cite le Ps 96,5: en hébreu, les deux versets sont identiques et ils ont l'expression élil. Cette forme, qui apparaît quasi exclusivement au pluriel, a toujours une valeur péjorative dans la Bible hébraïque et pourrait avoir été créée $a d$ hoc pour ce propos. Le nom rappelle l'adjectif 'ělill, «faible», et pourrait évoquer de manière polémique le substantif ' $e l$, « dieu »113. Or, d'après une logique que nous avons déjà vue à l'œuvre en Deut 32, dans le texte grec du Ps 95,5 (= 96,5 TM) 'élil est traduit par $\delta \alpha \iota o^{\prime} v \alpha$ :

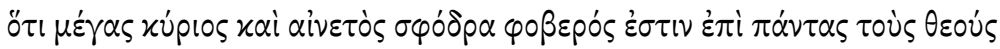

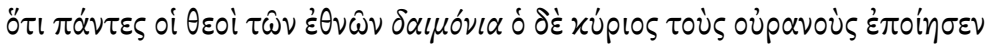

Par contre, en 1 Paral 16,26 le traducteur des livres des Chroniques choisit $\varepsilon \varepsilon^{\prime} \delta \omega \lambda \alpha$ pour le même référent hébreu :

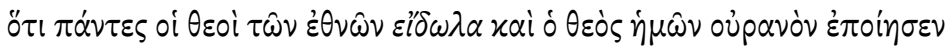

Une telle différence n'est pas nécessairement surprenante car 'élilim n'est jamais traduit de manière systématique dans la LXX. Au contraire, nous trouvons une solution différente presque à chaque occurrence. Néanmoins, tous les choix proposés reflètent le langage de la polémique contre les idoles : c'est

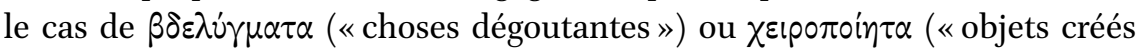
par l'homme ») $)^{114}$. En outre, l'équivalence entre $\varepsilon^{\prime \prime} \delta \omega \lambda \alpha$ et 'élilìm a un précédent

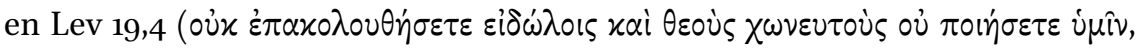

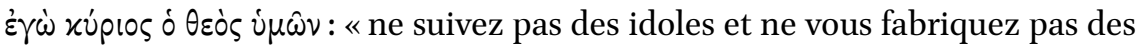
dieux sculptés, je suis le Seigneur votre dieu ») et il est possible qu'elle ait été influencée par les traditions sur le Décalogue. La comparaison entre le texte grec des Psaumes et celui des Chroniques démontre, en tout cas, la proximité entre la notion d' $\varepsilon^{\prime} \delta \omega \lambda$ ov et celle de $\delta \alpha \mu \rho \dot{v} v i o v$.

Pour sa part, la mention des $\varepsilon i \delta \omega \lambda \alpha$ nous ramène au contexte de la polémique contre lesdites « idoles » ainsi que vers l'interdiction des images divines, voire

113 Voir Preuss 1977.

114 Voir par exemple Is 2,8, 18, 20 ; 10,11; 19,1; 31,7. 
des images « tout court », telle qu'énoncée par le Décalogue, tout en soulevant la question de sa réception ancienne. Lorsque les traducteurs (et, par la suite, les auteurs) de la LXX construisent la polémique contre les dieux étrangers en les définissant comme $\varepsilon i \delta \omega \lambda \lambda$, ils ont recours à une idée qui, comme nous l'avons vu, peut parfois être associée à ce terme grec: celle d'une forme privée de réalité, d'un fantôme, qui met l'accent sur l'aspect de vacuité et de vanité et qui correspond bien aux notions exprimées en hébreu par 'ělilîm, hăbālîm, etc.

En y regardant d'un peu plus près, cet usage implique, néanmoins, un glissement important dans la sémantique grecque d' $\varepsilon 1 \delta \omega \lambda$ ov. Comme l'ont bien montré les études de Jean-Pierre Vernant ${ }^{115}$, l'aspect saillant de l'zîं $\omega \lambda$ ov grec n'est pas son inconsistance ni son caractère trompeur, qui est en revanche souligné dans certains textes bibliques. L'zî̉ $\omega \lambda$ ov est plutôt caractérisé par sa capacité à "présentifier » quelqu'un ou quelque chose. Il peut également, par sa fonction performative, servir d'équivalent fonctionnel de la personne ou de l'objet qui est représenté (et, en ce sens, il ne correspond que partiellement à notre concept moderne de «fantôme», qui reste une traduction peu précise).

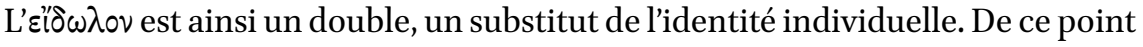
de vue, il renvoie au champ sémantique de l'image, conçue en tant que représentation visuelle, et il peut se référer autant aux images privées de réalité, que l'on qualifierait d'« apparences », qu'à des objets bien concrets comme, par exemple, des statues. Un des cas révélateurs de cette nature de l'zîं $\omega \lambda$ ov grec est constitué par la statue du guerrier troyen Protésilas, érigée par son épouse Laodamia, qu'elle vénérait en secret dans son lit, car l'image représentait l'équivalent fonctionnel du mari parti à la guerre, à savoir qu'elle le remplaçait lors

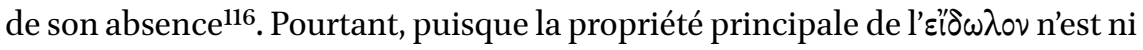
l'absence de réalité ni la ressemblance avec l'objet représenté, mais sa capacité à rendre visible une présence qui est ailleurs, la similarité n'est pas non plus une caractéristique essentielle. Ainsi, les kolossoi, ces pierres brutes levées comme cippes pour les morts, rentrent à plein titre dans la catégorie des $\varepsilon i \delta \delta \omega \lambda \alpha$. Par conséquent, la fonction de présentification qui est propre à l' $\varepsilon{ }^{\prime} \delta \omega \lambda$ ov rend ce terme parfait pour décrire les statues divines, images tangibles d'une réalité qui appartient à un monde invisible ou autre. Comme nous allons le voir, ce dernier aspect n'avait pourtant pas échappé aux traducteurs de la LXX.

\subsection{Les عi $\delta \omega \lambda \alpha$, entre images et idoles}

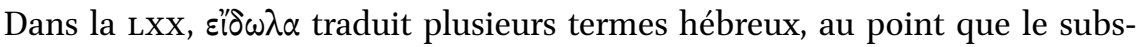
tantif semble dénoter une catégorie générale, celle d'«idole», qui réunit un

115 Vernant 1975, 1983, 199 (en réponse à Saïd 1987).

116 Pour l'analyse de cet épisode, voir Bettini 1992, p. 12-16. 
vocabulaire assez divers, toujours appliqué à des dieux illégitimes ou à des objets des cultes interdits. Il faut, toutefois, préciser que le concept d'idole ne naît pas avec les $\varepsilon i \delta \delta \nu \lambda \alpha$ de la LXX, mais qu'il est déjà opérationnel, dans une certaine mesure, dans la Bible hébraïque, même s'il n'est pas défini par une expression univoque. Par "polémique contre les idoles», on entend généralement une manière précise de critiquer les dieux étrangers, en les réduisant à de pures et simples images ${ }^{117}$. Cette polémique s'enracine dans les prohibitions du Décalogue qui interdisent aux Israélites de fabriquer des images (divines?) et de les vénérer comme si elles étaient des dieux. Cette interdiction est formulée dans les deux versions du Décalogue, en Ex 20,4 et en Deut 5,8. Ici, le terme $\varepsilon i \delta \omega \lambda$ ov apparaît pour la première fois comme équivalent de l'hébreu pesel. Il faut, toutefois, tenir compte du fait qu'en Deut 5,8 le codex Alexandrinus et le papyrus Chester Beatty (963) préservent la traduction plus littérale de pesel par $\gamma \lambda u ́ \pi \tau o v$, « objet sculpté », qui correspond vraisemblablement à la LXX ancienne ${ }^{118}$.

De manière générale, $\varepsilon^{\prime} \delta \omega \lambda$ ov compte environ soixante-dix occurrences dans la LXX dont douze se trouvent dans le Pentateuque. Il traduit quinze termes hébreux, se référant presque exclusivement à des dieux ou à des objets de culte étrangers ${ }^{119}$. Si l'on considère seulement le Deutéronome, l'on observe qu'हi̊̄ $\omega \lambda$ ov traduit au moins deux substantifs différents: hăbālîm en 32,21; gillûlim en 29,16 (et encore pesel en Deut 5,8 dans le Vaticanus).

Une hypothèse proposée par $F$. Büschel dans les années soixante, puis reprise par F. Barnes Tatum et encore acceptée aujourd'hui ${ }^{120}$, voit dans l'in-

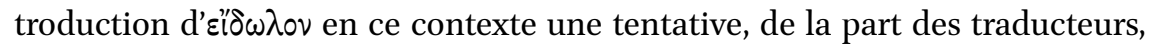
de réinterpréter le deuxième commandement comme anti-idolâtre, et non comme simplement anti-iconique comme il l'était à l'origine ${ }^{121}$. D'après cette hypothèse, la prohibition imposée par Yhwh aux Israélites telle qu'elle était comprise à l'époque des traducteurs ne concernerait pas toute image divine, et encore moins les représentations visuelles tout court, mais seulement les images des divinités étrangères, désormais dévalorisées et réduites à de simples «idoles » ${ }^{122}$. L'hypothèse s'appuie sur deux éléments : le fait que

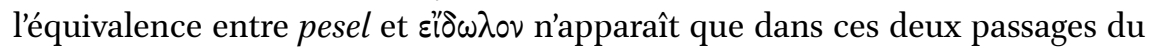

\footnotetext{
117 Voir Levtow 2008, 16-18; Ammann 2015, p. 12-13.

118 Cette lecture est donc préférée par Wevers 1977.

119 Avec l'exception des tĕrāpîm en Gen 31,19, 34, 35.

120 Büschel 1964; Barnes Tatum 1986.

121 Barnes Tatum 1986, p. 178-179.

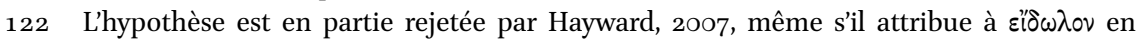
Ex 20,4 la signification d'« idole». Voir également la discussion en Barbu 2011 ; id. 2016, p. $40-51$.
} 
Décalogue et l'idée selon laquelle $\varepsilon{ }^{\prime} \delta \omega \lambda$ ov ne serait jamais utilisé pour désigner des images divines en dehors des traditions juives et chrétiennes. En outre, l'idée que la signification première d' $\varepsilon^{\prime} \delta \omega \lambda$ ov soit celle d'image «illusoire», et donc «fausse», serait renforcée par la connotation péjorative assignée à ce mot dans les écrits platoniciens. Dans la théorie de l'image et de la vision développée par Platon, d'abord dans Le Sophiste et ensuite dans La République, le philosophe oppose effectivement la fidélité del'zl'xwv à la nature trompeuse de l' $\varepsilon \imath^{\prime} \delta \omega \lambda{ }^{123}$.

Les interprétations de Büschel et de Barnes Tatum ont donc tendance à réduire les usages d' $\varepsilon^{\prime} \delta \omega \lambda$ ov dans la LXX à une logique univoque. Mais leur hypothèse n'est pas entièrement vérifiée. Dans un article de 2002, Terry Griffith a réuni une série de textes littéraires dans lesquels $\varepsilon^{\prime} \delta \omega \lambda$ ov désigne clairement des statues divines ou des objets de culte ${ }^{124}$. Outre un passage de Polybe assez bien connu ${ }^{125}$, parmi les auteurs repérés par Griffith figurent Hérodote et Ésope, et nombres d'auteurs d'époque hellénistique, tels que Diodore de Sicile, Denys d'Halicarnasse et Plutarque.

En outre, les données papyrologiques confirment les résultats mis en évidence par Griffith pour les textes littéraires et ouvrent de nouvelles pistes. Deux pétitions sont particulièrement intéressantes à ce sujet. Un papyrus grec démotique provenant de Tebt et datant de 87 av. n. è. contient une pétition rédigée par un certain Armisios et ses collègues qui travaillent comme embaumeurs d'ibis et de faucons dans le temple d'Hermès ${ }^{126}$. Armisios dénonce des malfaiteurs qui, s'étant introduits dans le temple alors qu'il était en train de laver les vêtements des dieux, l'ont frappé à plusieurs reprises et ont volé les habits divins. Pour décrire les images divines Armisios utilise les expressions

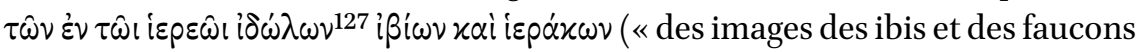

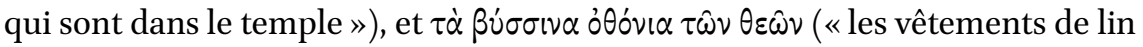

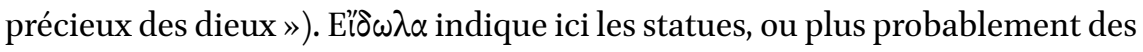
momies d'ibis et de faucons qui étaient censées représenter les dieux et qui étaient recouvertes de lin.

Dans la même ligne, un ostracon trouvé près du cimetière des poissons sacrés à Latopolis, datant du $\mathrm{I}^{\mathrm{er}}$ ou $\mathrm{II}^{\mathrm{e}}$ siècle de notre ère, contient une pétition oraculaire adressée à Athéna par des embaumeurs de béliers, Ammonios et

\footnotetext{
123 Pl., Sph. 240a-b ; R. 7, 516 sqq.

124 Griffith 2002.

125 Pol., Hist. 30, 25, 13-15, qui décrit les processions au village de Daphné, près d'Antioche, pour les jeux en l'honneur d'Antiochus IV.

126 PStrasb. 2 91, ed. Preisigke 1920. Pour le texte complet avec traduction anglaise des papyri ici discutés, voir Angelini 2019, p. 257-26o.

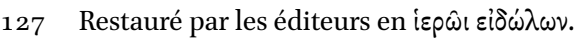


ses collègues, injustement accusés par leurs ennemis le jour de la fête dédiée

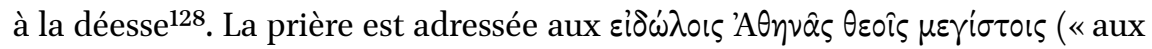
images sacrées d'Athéna, les grands dieux »), et une expression similaire apparait encore quelques lignes plus loin, lorsque les embaumeurs demandent

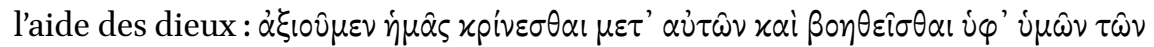

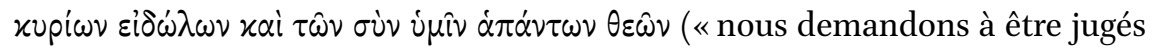
par vous et à recevoir de l'aide de votre part, images maîtres, et de la part de tous les dieux qui sont avec vous »). La question est donc de savoir à quoi ressemblaient ces images d'Athéna qui étaient sans doute des objets présents dans le temple et présumés aptes à représenter la déesse pour que la pétition soit efficace. Or, à Latopolis la déesse égyptienne Neith était identifiée à Athéna depuis longtemps; en outre, le poisson latos (lates niloticus) était considéré comme consacré à la déesse et faisait l'objet d'un culte. Il est donc probable que les $\varepsilon^{\prime} \delta \omega \lambda \alpha$ aient été des momies de poissons conservées dans le temple, qui fonctionnaient comme substituts d'Athéna-Neith. La formulation du papyrus met d'ailleurs en parallèle les $\varepsilon^{\prime} \delta \omega \lambda \alpha$ xúpı, les «images maîtres»- au sens où elles représentent la divinité principale du temple, et les autres dieux qui

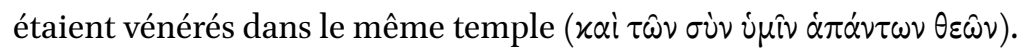

Cette interprétation est encore appuyée par un troisième papyrus de Tebt, du I ${ }^{\text {er }}$ siècle de notre ère. Il transcrit le serment d'une corporation de pêcheurs des villages de la région qui jurent de s'abstenir de la capture de l'oxyrhynque et du lépidote, deux poissons sacrés pour Osiris. Encore une fois, le texte uti-

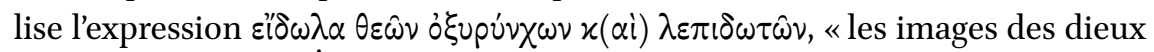
oxyrhynques et lépidotes», qui indique la proximité fonctionnelle entre les divinités et «leurs» poissons ${ }^{129}$.

Ces témoignages permettent, donc, de conclure que, dans la formulation du deuxième commandement dans la LXx d'Ex 20,4, $\varepsilon^{\prime \prime} \delta \omega \lambda$ ov ne doit pas être compris de manière abstraite ou polémique comme «idole». Au contraire, le terme suggère l'idée d'une image, probablement une statue, qui agit comme substitut du dieu et dont la fonction est de présentifier la divinité aux yeux du fidèle $^{130}$. Dans cette perspective, le choix opéré par le traducteur de l'Exode relève d'une réception spécifique du deuxième commandement: un résultat

128 P.Worp 7 (= O. Garstand 1), ed. Boyaval 1980 ; Gallazzi 1985; Gascou 2008 avec traduction française. Le contenu de l'accusation n'a pas été préservé. Sur les pétitions oraculaires comme genre littéraire, voir Versnel 1991.

129 Hunt et Edgar, Sel. Pap. 2, no. 329, Loeb 1934.

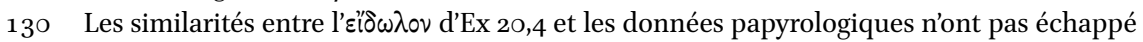
à l'observation minutieuse de James Aitken qui, dans un article de 2014, suggérait que la compréhension d' $\varepsilon i \delta \omega \lambda$ ov comme «image » était la lecture la plus simple du passage (p. 193). Voir également Le Boulluec et Sandevoir 1989, p. 205-206. 
qui n'est pas étonnant lorsque l'on tient compte du fait que l'interdiction sur les images était susceptible d'interprétations différentes encore aux époques hellénistique et romaine. À cet égard, il suffit de comparer les interprétations proposées par Josèphe, d'après qui la reproduction de tout être vivant ( $\mu \eta \delta \varepsilon v o ̀ s$

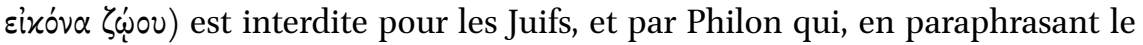
deuxième commandement, utilise le langage propre des statues divines $(\pi \varepsilon p i$

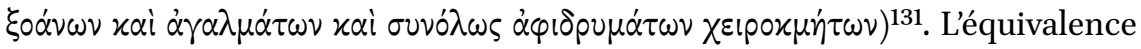
entre pesel et $\varepsilon$ "í $\omega \lambda$ ov apparaît, dès lors, comme parfaitement respectueuse à la fois de la langue source (l'hébreu) et de la langue cible (le grec).

En conclusion de ce long parcours, nous pouvons, donc, tirer quelques réflexions générales sur la manière dont la catégorie de démon est mobilisée pour penser les dieux des autres. Nous avons vu que le Chant de Moïse met pour la première fois en place l'idée des démons comme puissances étrangères à la cour céleste, inférieures à Yhwh et inconciliables avec sa souveraineté et, par conséquent, avec son culte. Une telle notion n'est pas introduite par les traducteurs, mais est déjà opérationnelle dans la forme pré-massorétique du texte hébreu. De ce point de vue, la LXx démontre une continuité considérable avec son texte source, tout en s'alignant également avec d'autres traditions du

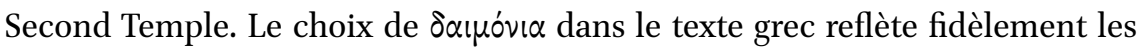
šédîm de sa Vorlage. Tout en insistant sur l'opposition entre les démons et la divinité, le texte encourage la représentation des $\delta \alpha \mu o ́ v i \alpha$ comme puissances potentiellement négatives qui n'appartiennent pas au panthéon traditionnel et qui sont clairement distinguées non seulement de Yhwh, mais également des autres membres de la cour céleste, notamment des anges. D'autres textes de la LXX qui insistent sur la même thématique, comme les Ps 95 et 105, les Odes, le livre de Baruch et, probablement, Is 65,3 prolongent cette opposition. Le rapprochement entre ces textes et d'autres matériels grecs contemporains confirme, non seulement, qu'une telle représentation s'inscrit parfaitement dans le contexte culturel et religieux de l'époque qui tend à concevoir les démons comme des puissances inconsistantes, inférieures et subordonnées aux dieux, mais atteste, également, le fait que la LXX représente

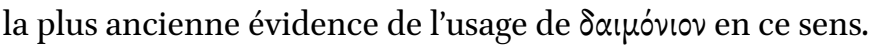

En outre, la comparaison des parallèles grecs repérés chez Platon et Plutarque avec les textes bibliques montre les éléments de nouveauté qui sont propres à ces derniers. La spécificité biblique réside, surtout, dans le lien étroit qui se développe entre la critique des démons et la polémique contre les idoles, au point que les deux peuvent être considérés comme des fautes cultuelles fonctionnellement équivalentes.

131 Jos., AJ 3, 91 ; Philo, Dec. 51. 
Enfin, du point de vue sémantique, le répertoire d'occurrences ainsi ana-

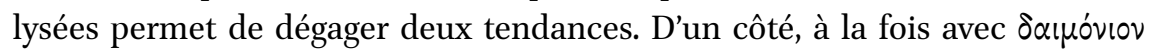
et avec $\varepsilon i \delta \omega \lambda$ ov, les traducteurs ont tendance à sélectionner un signifié spécifique à l'intérieur de deux spectres polysémiques très larges. Le succès des équivalences choisies par les traducteurs grecs contribue à figer une signification univoque pour $\delta \alpha \iota \mu o ́ v i \alpha$ et $\varepsilon i \delta \omega \lambda \alpha$ dans la LXX, et ce choix aura un impact considérable sur les traditions successives. De l'autre côté, nombre d'indices suggèrent que la polysémie de ces notions est encore bien présente à l'esprit des traducteurs grecs (au moins à certains d'entre eux) ${ }^{132}$. Pour ce qui concerne spécifiquement les $\delta \alpha \mu o_{v i} \alpha$, les deux occurrences de la LXX d'Is 65, qui font vraisemblablement référence à des rituels domestiques de détournement pour les démons et au culte du «Bon démon » et de la «Bonne Fortune », sont particulièrement significatives à cet égard. Lorsque l'on combine ces résultats avec ceux mis en évidence dans le chapitre précédent pour la LXX d'Is 13 et 34, mettant en scène un contexte différent où les $\delta \alpha \iota \mu o ́ v i \alpha$ étaient insérés à l'intérieur du paysage naturalisé des régions exotiques, on acquiert la mesure de la versatilité religieuse et culturelle de cette notion auprès du traducteur du livre d'Isaïe.

132 Quant à la naissance du concept d'idole, mon analyse confirme et précise certaines des conclusions déjà mises en évidence par les travaux de Daniel Barbu (Barbu 2011 ; id. 2016, p. 51). 